\title{
Interventional 4-D C-arm CT Perfusion Imaging Using Interleaved Scanning and Partial Reconstruction Interpolation
}

\author{
Andreas Fieselmann, Arundhuti Ganguly, Yu Deuerling-Zheng, Michael Zellerhoff, Christopher Rohkohl, \\ Jan Boese, Joachim Hornegger, Member, IEEE, Rebecca Fahrig
}

\begin{abstract}
Tissue perfusion measurement during catheterguided stroke treatment in the interventional suite is currently not possible. In this work, we present a novel approach that uses a $\mathrm{C}$-arm angiography system capable of CT-like imaging (C-arm CT) for this purpose. With $\mathrm{C}$-arm $\mathrm{CT}$ one reconstructed volume can be obtained every 4-6 $\mathrm{s}$ which makes it challenging to measure the flow of an injected contrast bolus. We have developed an interleaved scanning (IS) protocol that uses several scan sequences to increase temporal sampling. Using a dedicated 4-D reconstruction approach based on partial reconstruction interpolation (PRI) we can optimally process our data.

We evaluated our combined approach (IS-PRI) with simulations and a study in 5 healthy pigs. In our simulations, the cerebral blood flow values (unit: $\mathrm{ml} / 100 \mathrm{~g} / \mathrm{min}$ ) were 60 (healthy tissue) and 20 (pathological tissue). For one scan sequence the values were estimated with standard deviations of 14.3 and 2.9, respectively. For two interleaved sequences the standard deviations decreased to 3.6 and 1.5, respectively. We used perfusion CT to validate the in vivo results. With two interleaved sequences we achieved promising correlations ranging from $r=0.63$ to $r=0.94$. The results suggest that $\mathrm{C}$-arm $\mathrm{CT}$ tissue perfusion imaging is feasible with two interleaved scan sequences.
\end{abstract}

Index Terms-Perfusion imaging, dynamic reconstruction, C-arm CT, stroke treatment.

\section{INTRODUCTION}

$\mathbf{S}$ TROKE is a serious medical emergency and a leading cause of death and disability world-wide. During an ischemic stroke event the blood supply to the brain is locally disrupted and fast treatment is mandatory. Perfusion CT and MRI are the common imaging modalities used to identify therapeutic options for potentially salvageable tissue [1]. However, these modalities are not available in the interventional suite where catheter-guided stroke therapy procedures, such as intra-arterial thrombolysis, actually take place. Interventional

A. Fieselmann (e-mail: andreas.fieselmann@informatik.uni-erlangen.de) and J. Hornegger are with the Department of Computer Science, Pattern Recognition Lab, and the Erlangen Graduate School in Advanced Optical Technologies (SAOT), Friedrich-Alexander University of ErlangenNuremberg, Martensstr. 3, 91058 Erlangen, Germany

A. Ganguly and R. Fahrig are with the Department of Radiology, Lucas MRS Center, Stanford University, 1201 Welch Road, Palo Alto, CA 94305

A. Fieselmann, Y. Deuerling-Zheng, M. Zellerhoff, C. Rohkohl and J. Boese are with Siemens AG, Healthcare Sector, Angiography \& Interventional X-Ray Systems, Siemensstr. 1, 91301 Forchheim, Germany

The concepts and information presented in this paper are based on research and are not commercially available.

Copyright (c) 2012 IEEE. Personal use of this material is permitted. However, permission to use this material for any other purposes must be obtained from the IEEE by sending a request to pubs-permissions@ieee.org. tissue perfusion measurement immediately before starting the procedure could be useful because the state of perfusion can change after the initial diagnosis. It may also be useful during the procedure to determine the treatment success and treatment endpoint [2]. In order to enhance interventional stroke therapy we have therefore developed a novel approach that uses a $\mathrm{C}$-arm angiography system capable of CT-like imaging (C-arm CT, Figure 1) [3] for interventional tissue perfusion imaging.

In perfusion CT a contrast bolus is injected and several reconstructed 3-D volumes are obtained at short (typically $1 \mathrm{~s}$ ) intervals in order to measure the contrast flow in each voxel individually. Various perfusion parameters such as cerebral blood flow (CBF) and volume (CBV), mean transit time (MTT) and time-to-peak (TTP) are computed from the reconstructed time-attenuation curve at each voxel position [1].

The main challenge for measuring these parameters with $\mathrm{C}$-arm CT is the relatively slow $\mathrm{C}$-arm rotation speed which, for example, limits the temporal sampling (typically one sample every 4-6 s) of the reconstructed time-attenuation curves.

To date, there has been little work on image reconstruction for dynamic C-arm CT perfusion imaging. An iterative reconstruction algorithm to compensate for the effects of the slow C-arm rotation speed has been presented in [4]. However, the high dimensionality of the proposed optimization leads to long computation times for the large volume data sets which would make this approach currently not feasible for use with real data during interventional stroke therapy.

Feldkamp-type algorithms, which are based on the analytical filtered backprojection (FBP) reconstruction approach, can be implemented such that they are computationally very fast [5], [6]. In [7] a method for perfusion imaging with a slower continuously rotating CT scanner based on a dynamic Feldkamp-type reconstruction algorithm has been presented. The motivation for this method was to reduce X-ray dose by operating the CT scanner at a slow rotation speed.

In this paper, we will present our novel combined approach for C-arm CT perfusion measurement, which we termed IS-PRI (interleaved scanning - partial reconstruction interpolation), that is a combination of a specialized scanning protocol and a dedicated reconstruction method. The scanning protocol uses several, interleaved C-arm CT acquisitions which can be optimally processed by our 4-D interpolation-based reconstruction method. Similar to the work in [7] our reconstruction method is also a dynamic Feldkamp-type algorithm which 
makes it computationally fast.

This paper is an extension of previous work [8]. Compared to [8], we described our methods in more detail, performed new numerical simulations and validated our methods more extensively using in vivo data. Results based on our methods were also recently presented in [9] with a focus on their clinically relevant aspects. The focus of this paper is now to provide a detailed technical description and investigation of these methods.

Our paper is organized as follows: In Section II, we will explain the technical challenges in C-arm CT perfusion imaging and give a detailed description of our methods. We will investigate these methods using simulated data in Section III and validate its feasibility with real data using an in vivo study in a healthy pig model in Section IV. Potential future developments based on new C-arm CT systems or reconstruction approaches are outlined in Section V. In Section VI, we will give a summary and conclusion.

\section{SPecialized Scanning Protocol and RECONSTRUCTION METHOD}

In this section, we will first explain the technical challenges that have been identified in order to implement CTperfusion-like imaging with a $\mathrm{C}$-arm CT. Then, two approaches will be presented, a specialized scanning protocol and an interpolation-based 4-D reconstruction method, which are the technical solutions that we have developed.

\section{A. Challenges in C-arm-CT-Based Perfusion Imaging}

1) Low Temporal Sampling: We will start with a description of 4-D imaging with a C-arm CT. In order to obtain $N_{\text {rot }}$ reconstructed 3-D volumes the C-arm has to be rotated $N_{\text {rot }}$ times, each time through approximately $200^{\circ}$, in a bidirectional manner. Note, current $\mathrm{C}$-arm CT systems are not capable of continuous, uni-directional C-arm rotations. During each rotation 2-D projection data is acquired. The time period for one rotation (typically $3-5 \mathrm{~s}$ ) will be denoted $T_{\text {rot }}$. A short waiting time $T_{\mathrm{w}}$ (typically $1 \mathrm{~s}$ ) is required between two rotations. The middle plot in Figure 2(a) shows the viewangle $\lambda(t) \in[0, \Lambda]$ of such a multi-rotational scan sequence plotted against time $t$. A multi-rotational scan sequence is used in cardiac C-arm CT with retrospective electrocardiogram (ECG)-gating, for example [10].

In perfusion CT the sample period is typically $1 \mathrm{~s}$ but it may be increased to up to $3 \mathrm{~s}$ and similar diagnostic quality of the computed perfusion parameter maps may still be provided [11], [12]. However, in perfusion imaging with a C-arm CT the temporal sample period, given by $T_{\text {rot }}+T_{\mathrm{w}}$, obtained with such a 4-D scan protocol, is typically longer than $3 \mathrm{~s}$ and may not be sufficient for adequate sampling of the reconstructed time-attenuation curve. In particular, arterial time-attenuation curves, which are used for normalization of the perfusion values [13], have relatively fast contrast dynamics and may be undersampled [12].

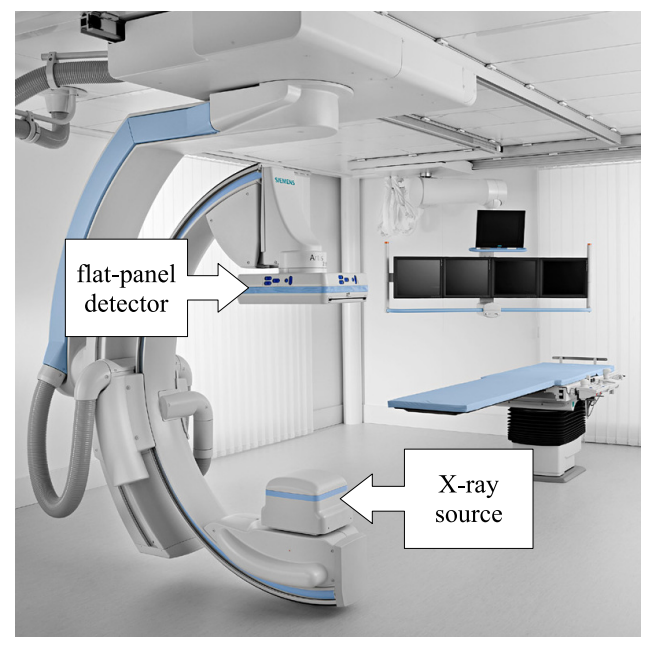

Fig. 1. C-arm angiography system (Artis zee ceiling-mounted system, Siemens AG, Healthcare Sector, Forchheim, Germany) capable of CT-like imaging in the interventional suite.

2) Inconsistent Projection Data: The acquired projection data is inconsistent due to the (intentional) time-varying contrast concentration in the region of interest during one $\mathrm{C}$-arm rotation. We will describe two kinds of FBP reconstruction artifacts that can occur. In order to explain these artifacts we will consider one single point object with time-varying attenuation values that we approximate by a discrete voxel. Note, due to the linearity of the FBP our explanations can be easily generalized to an arbitrary number of voxels with time-varying attenuation values.

First, the reconstructed time-attenuation curve measured at this voxel position is not a sampled version of the true time-attenuation curve. In fact, the relatively long acquisition process leads to a low-pass filtering of the true curve. By investigation of the FBP algorithm it can be shown that this process can be approximated by a convolution of the true curve with a rectangular function of temporal width $T_{\text {rot }}$ before it is sampled [7]. Due to the low-pass filtering the peak value of a reconstructed time-attenuation curve will be underestimated even if the sample time point, i.e. the central time of the $\mathrm{C}$-arm rotation, coincides with the time of the peak value of the curve.

Second, streak artifact in the reconstructed 3-D volumes can appear around the voxel with time-varying attenuation values. A detailed description of this kind of artifact is given in [14]. An example from a numerical simulation is shown in Figure 8(a). The magnitude of this kind of artifact depends on the slope of the time-attenuation curve during the $\mathrm{C}$-arm rotation. Therefore, this artifact will be most prominent around arterial vessels in which the attenuation values change rapidly and thus have a high slope.

\section{B. Interleaved Scanning (IS)}

In order to improve the low temporal sampling (see Section II-A1) we propose an interleaved scanning protocol with $N_{\text {seq }}$ different multi-rotational sequences. Each of the $N_{\text {seq }}$ multi-rotational sequences consists of $N_{\text {rot }}$ C-arm rotations, each of which provides a full set of projection data. In total 

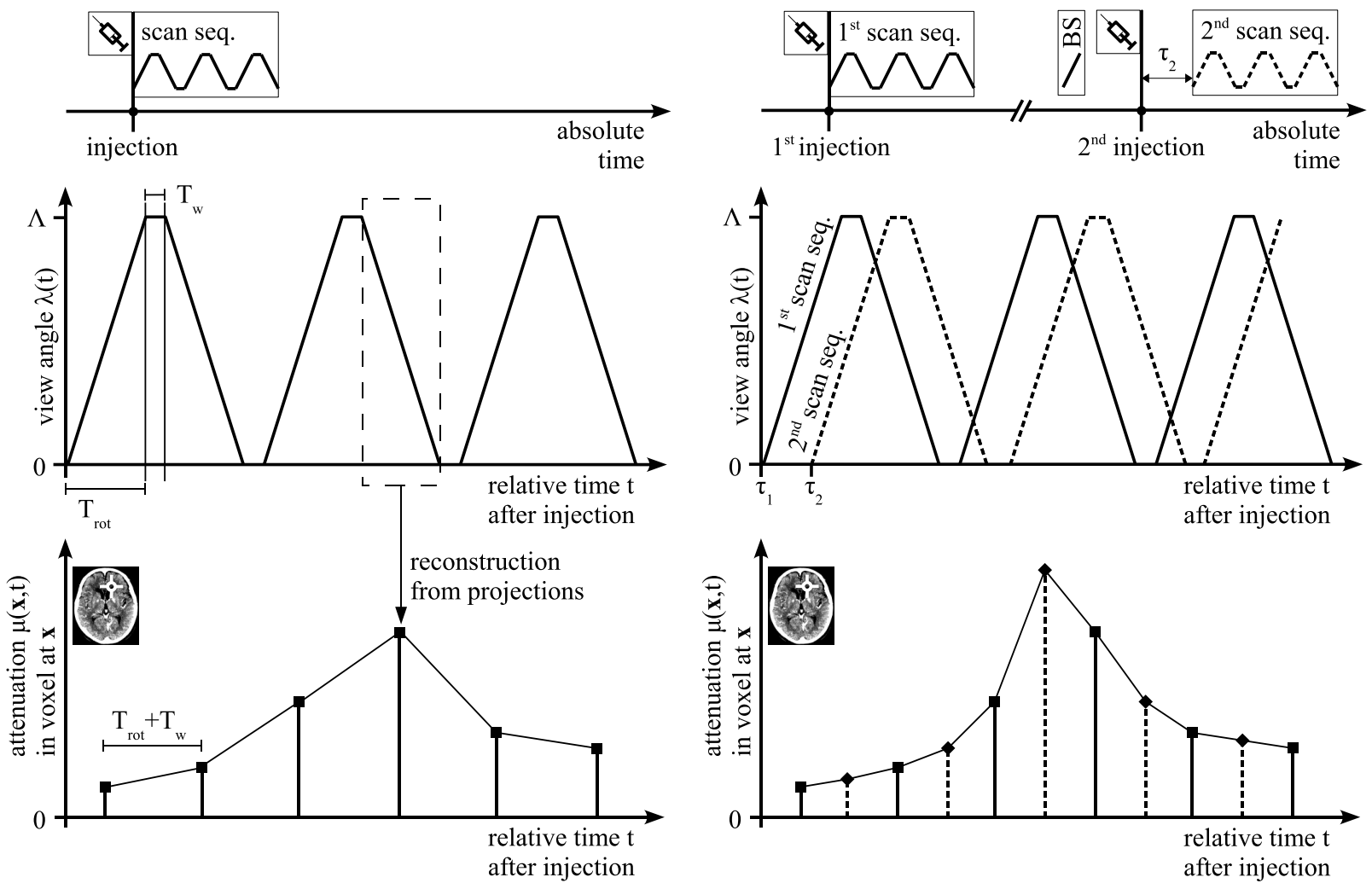

(a) One scan sequence $\left(N_{\text {seq }}=1\right)$

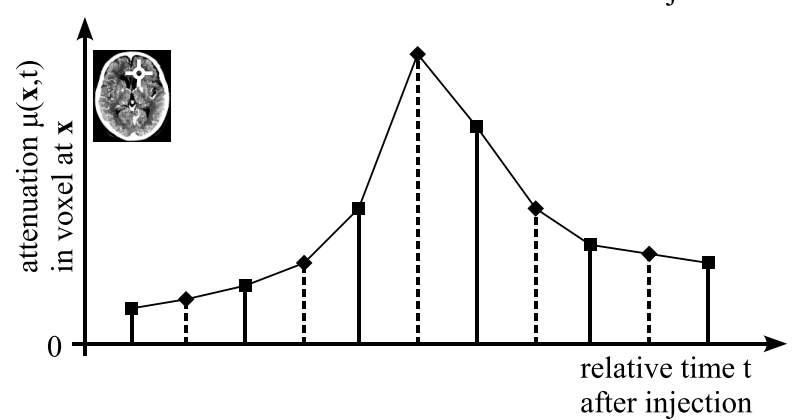

(b) Two interleaved scan sequences $\left(N_{\text {seq }}=2\right)$

Fig. 2. The left figure shows the case with one scanning sequence whereas the right figure shows the principle of interleaved scanning where two scan sequences have different delay times $\tau_{1}$ and $\tau_{2}$ relative to the time of injection. Top row: The timing of the injections, the scan sequences, and the additional baseline scan (BS; only relevant for interleaved scanning) is shown on an absolute time scale. Middle row: During each scan sequence the X-ray source of the C-arm CT system rotates in a bi-directional manner; the scan sequences are shown on a relative time scale after the time of injection. Bottom row: One volume is reconstructed from the data of each rotation and for each voxel a time series can be measured.

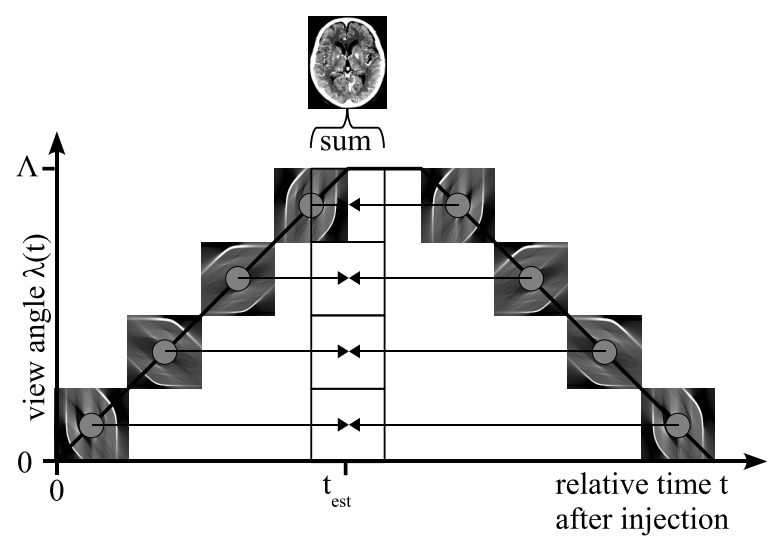

(a) One scan sequence $\left(N_{\text {seq }}=1\right)$

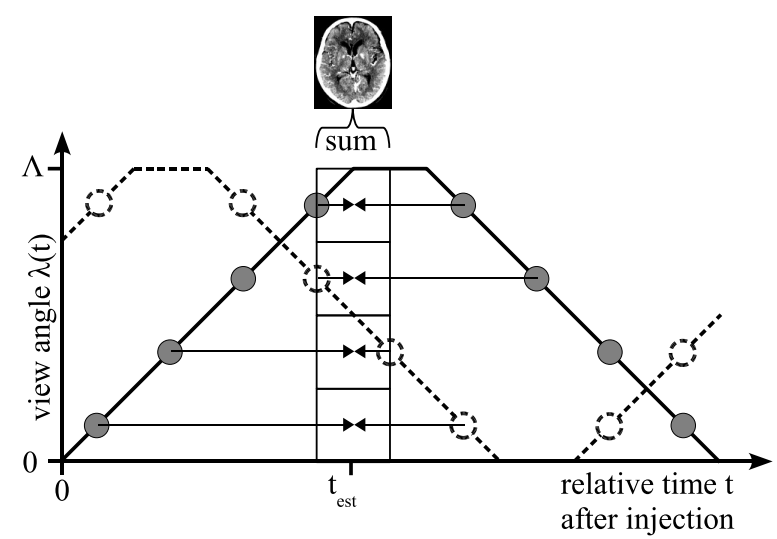

(b) Two interleaved scan sequences $\left(N_{\text {seq }}=2\right)$

Fig. 3. Partial reconstruction interpolation with $M=4$ angular interpolation intervals to create a reconstructed volume at $t=t_{\text {est }}$. In the right figure the data from two interleaved scans is actually combined to yield better interpolation results. 
$N_{\text {seq }} \times N_{\text {rot }}$ datasets are acquired. The interleaved scanning protocol has two important features. First, a new bolus with identical injection parameters will be injected before each multi-rotational sequence. Second, for each multi-rotational sequence there will be a different delay time between the start of the bolus injection and the start of the scanning.

This interleaved scanning approach increases the sampling density of the projection data space and consequently the sampling density of the reconstructed time-attenuation curves extracted from each voxel. Figure 2(b) shows an example with two multi-rotational sequences.

We can determine the temporal delay $\tau_{n}$ between the start of the injection and start of the $n$-th multi-rotational sequence $\left(n=0, \ldots, N_{\text {seq }}-1\right)$ by

$$
\tau_{n}=\left(T_{\text {rot }}+T_{\mathrm{w}}\right) \times \frac{n}{N_{\mathrm{seq}}}+t_{\mathrm{c}}
$$

where $t_{\mathrm{c}}$ is a constant temporal offset. This definition of $\tau_{n}$ leads to a regular sampling of the central view-angle $\Lambda / 2$. Other definitions of $\tau_{n}$ with non-uniform sampling periods of $\Lambda / 2$, for example, are also possible. For $t_{\mathrm{c}} \leq-T_{\text {rot }}$ one or several baseline volumes without contrast agent are acquired before the acquisition of the contrast-enhanced volumes.

In perfusion imaging the enhancement (due to the contrast flow) above the baseline is analyzed to compute the perfusion parameters [13]. Therefore at least one baseline volume is needed which will then be subtracted from the following contrast-enhanced volumes. Thus, a practical choice would be $t_{\mathrm{c}}=-T_{\text {rot }}$. Note however, the baseline volume acquired at the beginning of the first sequence $(n=0)$ cannot be used as the baseline for later sequences $(n \geq 1)$ due to residual contrast from the previous bolus injections. In Section II-C the removal of this residual contrast will be discussed.

A method that is similar to our interleaved scanning approach was recently applied to lung perfusion imaging in rodents using a micro-CT [15]. The authors used several consecutive small volume injections of iodinated contrast agent, performed at a series of different starting view-angles, in order to increase the temporal sampling density.

\section{Partial Reconstruction Interpolation (PRI)}

With interleaved scanning as described in Section II-B we can increase the temporal sampling density of the perfusion time-attenuation curves. Nevertheless, the projection data for each reconstructed 3-D volume is acquired over a time interval $T_{\text {rot }}$. In order to compensate for the inconsistent projection data, and to reduce the two kinds of artifacts that were described in Section II-A2, we propose an interpolation-based 4-D reconstruction approach. It is motivated by a method that was previously investigated using a slowly-rotating CT scanner [7]. We have adapted this method to our C-arm CT scanning approach which, in contrast to the method in [7], uses several interleaved sequences each having alternating directions of Carm rotations. In order to describe our reconstruction approach we will first consider only one multi-rotational scan sequence, i.e. $N_{\text {seq }}=1$. We will then describe the case with multiple scan sequences.
We have $N_{\text {views }}$ different view-angles, starting at $\lambda_{0}$, with angular intervals $\Delta \lambda$. For each individual view-angle $\lambda_{l}$,

$$
\lambda_{l}=\lambda_{0}+l \times \Delta \lambda \quad\left(l=0, \ldots, N_{\text {views }}-1\right),
$$

we acquire a discrete sequence of cone-beam projections $p_{l, k}(u, v)$, where $u$ and $v$ are the usual coordinates on the planar detector, at time points $t_{l, k}\left(0 \leq k \leq N_{\text {rot }}-1\right)$ that refer to the time when the projection under the angle $\lambda_{l}$ was acquired during the $k$-th rotation.

In order to estimate an unknown projection value $\tilde{p}_{l, t_{\text {est }}}(u, v)$ at a certain time point $t_{\text {est }}$ with $t_{l, 0} \leq t_{\text {est }} \leq$ $t_{l, N_{\text {rot }}-1}$ we can apply temporal interpolation using the known projection values:

$$
\tilde{p}_{l, t_{\text {est }}}(u, v)=\sum_{k=0}^{N_{\text {rot }}-1} p_{l, k}(u, v) \varphi_{l, k}\left(t_{\text {est }}-t_{l, k}\right) .
$$

Possible interpolation functions $\varphi_{l, k}: \mathbb{R} \mapsto \mathbb{R}$ for nonuniformly sampled projection data, caused by the alternating directions of $\mathrm{C}$-arm rotations, will be discussed in Section II-D. In order to approximate a consistent data set we can apply this interpolation to estimate projections for all necessary combinations of $l, u$ and $v$ at a certain time point $t_{\text {est }}$.

However, during $\mathrm{C}$-arm $\mathrm{CT}$ scanning the projections may not be acquired at exactly the same angular position in forward and reverse rotations due to limited accuracy of the mechanical $\mathrm{C}$-arm motion. This would negatively impact the projectionbased interpolation. To take this into account and to increase the computational speed, the interpolation can also be applied using partially backprojected volumes. In order to describe this concept we will start by denoting the projection data of the $k$-th $\mathrm{C}$-arm rotation as

$$
\begin{array}{r}
\mathcal{P}_{k}=\left\{p_{0, k}\left(u_{\min }, v_{\min }\right), \ldots, p_{0, k}\left(u_{\max }, v_{\max }\right), \ldots\right. \\
\left., p_{N_{\text {views }}-1, k}\left(u_{\min }, v_{\min }\right), \ldots, p_{N_{\text {views }}-1, k}\left(u_{\max }, v_{\max }\right)\right\}
\end{array}
$$

where the subscripts min and max indicate the minimum and maximum detector coordinate, respectively.

We introduce the operator $\operatorname{PFBP}_{l_{1}}^{l_{2}}(\boldsymbol{x})\left\{\mathcal{P}_{k}\right\}$ for partial filtered backprojection in order to partially reconstruct an attenuation value at position $\boldsymbol{x}$ using only the projections from $\mathcal{P}_{k}$ with $l \in\left[l_{1}, l_{2}\right]$. This operator includes all steps of a normal FBP reconstruction, including the fan-beam redundancy weighting, for example, but backprojects the data over a limited angular interval only.

The PFBP operator can be implemented using a Feldkamptype algorithm [5] as

$$
\begin{aligned}
\operatorname{PFBP}_{l_{1}}^{l_{2}}(\boldsymbol{x})\left\{\mathcal{P}_{k}\right\} & =\sum_{l=l_{1}}^{l_{2}} \frac{m_{\Lambda}\left(\lambda_{l}, \hat{u}_{\lambda_{l}}(\boldsymbol{x})\right)}{w_{\lambda_{l}}(\boldsymbol{x})^{2}} \\
& \times \hat{p}_{l, k}\left(\hat{u}_{\lambda_{l}}(\boldsymbol{x}), \hat{v}_{\lambda_{l}}(\boldsymbol{x})\right) .
\end{aligned}
$$

Here, the functions $\hat{u}_{\lambda_{l}}(\boldsymbol{x})$ and $\hat{v}_{\lambda_{l}}(\boldsymbol{x})$ give the detector coordinates where a beam from the view-angle $\lambda_{l}$ passing through $\boldsymbol{x}$ intersects the detector, $m_{\Lambda}\left(\lambda_{l}, u\right)$ is a fan-beam redundancy weighting function [16] and $w_{\lambda_{l}}(\boldsymbol{x})$ is a distance weighting function, see [6] for details. The function $\hat{p}_{l, k}(u, v)$ 
is a bi-linear interpolation of the pre-processed (e.g., rampfiltered) projection $p_{l, k}(u, v)$.

Illustrations for these partial backprojections are shown in Figure 3(a). An important property of the PFBP operator is that a normal reconstruction of the value $\mu_{\mathrm{rec}}(\boldsymbol{x}, k)$ at $\boldsymbol{x}$ during the $k$-th $\mathrm{C}$-arm rotation, here assuming a time-independent object, is given by the sum of all partial filtered backprojections, i.e.

$$
\mu_{\text {rec }}(\boldsymbol{x}, k)=\sum_{j=0}^{M-1} \operatorname{PFBP}_{j L}^{(j+1) L-1}(\boldsymbol{x})\left\{\mathcal{P}_{k}\right\},
$$

where $L=N_{\text {views }} / M$ and $M$ is the number of angular interpolation intervals. In general, the number $L$ of viewangles per partial backprojection could also be non-uniform and angular windowing functions could be applied. Note, our partial reconstruction interpolation approach is generic and can also be based on reconstruction algorithms other than the FDK algorithm as long as these algorithms fulfill the condition in (6).

We can now apply the interpolation to the partial backprojections in order to reconstruct a value $\tilde{\mu}_{\text {rec }}\left(\boldsymbol{x}, t_{\text {est }}\right)$ corresponding to the time point $t_{\text {est }}$ :

$$
\begin{aligned}
\tilde{\mu}_{\text {rec }}\left(\boldsymbol{x}, t_{\text {est }}\right)= & \sum_{j=0}^{M-1} \sum_{k=0}^{N_{\text {rot }}-1} \operatorname{PFBP}_{j L}^{(j+1) L-1}(\boldsymbol{x})\left\{\mathcal{P}_{k}\right\} \\
& \times \varphi_{(j+0.5) L, k}\left(t_{\text {est }}-t_{(j+0.5) L, k}\right) .
\end{aligned}
$$

Next we will describe the use of this approach with data from an interleaved scanning protocol, i.e. $N_{\text {rot }} \geq 2$. In order to optimize the accuracy of the interpolation we will combine the data from different multi-rotational sequences. Mathematically, we change the summation endpoint of the inner sum in (7) from $N_{\text {rot }}-1$ to $N_{\text {rot }} \times N_{\text {seq }}-1$ and interpret $k$ as an index that can refer to $\mathrm{C}$-arm rotations from different multirotational sequences. This combined interleaved scanning (IS) and partial reconstruction interpolation (PRI) approach is also illustrated in Figure 3(b). The combination of both methods (IS-PRI) increases the temporal sampling density and can yield better approximations for consistent projection data sets.

In the IS-PRI approach we assume the change of contrast concentration in an projection interval of length $L$ to be negligible and the contrast-induced temporal enhancement function to be sufficiently smooth between two data time points in order to obtain accurate interpolation results.

As already mentioned in Section II-B, the Hounsfield values of the reconstructed $n$-th $\left(n=0, \ldots, N_{\mathrm{seq}}-1\right)$ scan sequence are influenced by residual contrast from the preceding $n$ bolus injections. Therefore, the data of each scan sequence $(n \geq 1)$ having $N_{\text {rot }}$ C-arm rotations is pre-processed in order to remove the attenuation values due to the residual contrast. Before each scan sequence $(n \geq 1)$ a new baseline scan is acquired (Figure 2(b)(top)) and reconstructed to provide $M$ partial backprojection baseline images. Then this baseline data is subtracted, individually for each of the $M$ intervals, from the partial backprojection data of the following $N_{\text {rot }}$ rotations. Finally the baseline acquired at the start of the 0-th scan sequence is again added to this partial backprojection data individually for each of the $M$ intervals.
TABLE I

SCAN PARAMETERS FOR THE NUMERICAL SIMULATIONS AND THE IN VIVO STUDY.

\begin{tabular}{lll}
\hline & $\begin{array}{l}\text { parameter set 1 } \\
\text { (simulations) }\end{array}$ & $\begin{array}{l}\text { parameter set 2 } \\
\text { (in vivo study) }\end{array}$ \\
\hline view-angle increment & $0.5^{\circ}$ & $1^{\circ}$ \\
number of views per rotation & 401 & 191 \\
angular range per rotation $(\Lambda)$ & $200^{\circ}$ & $190^{\circ}$ \\
time per rotation $\left(T_{\text {rot }}\right)$ & $4.30 \mathrm{~s}$ & $4.30 \mathrm{~s}$ \\
time between rotations $\left(T_{\mathrm{w}}\right)$ & $1.25 \mathrm{~s}$ & $1.25 \mathrm{~s}$ \\
number of rotations $\left(N_{\text {rot }}\right)$ & 9 & 6 \\
total scanning time & $48.7 \mathrm{~s}$ & $32.05 \mathrm{~s}$ \\
source-to-isocenter distance & $800 \mathrm{~mm}$ & $785 \mathrm{~mm}$ \\
source-to-detector distance & $1200 \mathrm{~mm}$ & $1198 \mathrm{~mm}$ \\
detector pixel size & $0.6 \times 0.6 \mathrm{~mm}^{2}$ & $0.616 \times 0.616 \mathrm{~mm}^{2}$ \\
number of detector pixels & $800 \times 1$ & $616 \times 480$ \\
& $($ no binning $)$ & $\left(\right.$ after $\left.4 \times 4 \mathrm{binning}^{\circ}\right)$ \\
total detector size & $480 \times 0.6 \mathrm{~mm}^{2}$ & $\approx 380 \times 296 \mathrm{~mm}^{2}$ \\
\hline
\end{tabular}

This procedure adds $N_{\mathrm{seq}}-1$ additional baseline scans, i.e. one for each interleaved sequence, to the overall number of scans. The additional baseline scans could actually be part of the multi-rotational sequence or acquired separately before that sequence. The latter may be prefered if the number of scans in a multi-rotional sequence is limited by hardware constraints, for example, in order to avoid temporal truncation of the timeattenuation curves.

\section{Interpolation of Non-Uniformly Sampled Data}

Due to the alternating directions of $\mathrm{C}$-arm rotations the projection data is sampled using non-uniform time intervals. This has to be considered when choosing suitable interpolation functions $\varphi_{l, k}$ in (7). There are various interpolation methods for non-uniformly sampled data and we will provide an overview of 5 different methods.

Nearest neighbor (NN) and linear (LIN) interpolation are robust against noisy data and they preserve the monotonicity of the data that is being interpolated. I.e. the interpolated values between two adjacent sample points are either strictly increasing or strictly decreasing. Cubic spline (CS) interpolation generates smooth curves which can be non-monotonic but it can lead to unintended overshoots or undershoots when interpolating noisy data [17]. Another class of interpolation methods are piecewise cubic Hermite interpolating polynomials (HIP) [18]. They also preserve the monotonicity of the data but they generate smoother curves when compared to linear interpolation. Radial basis function (RBF) interpolation computes an interpolated value by using a distance-dependent weighting of the known sample values [19]. For example, in [10] a Gaussian function has been used as weighting function.

\section{NUMERICAL SimULATIONS}

Numerical simulations were performed to investigate the properties of the acquisition and reconstruction scheme described in Section II. 


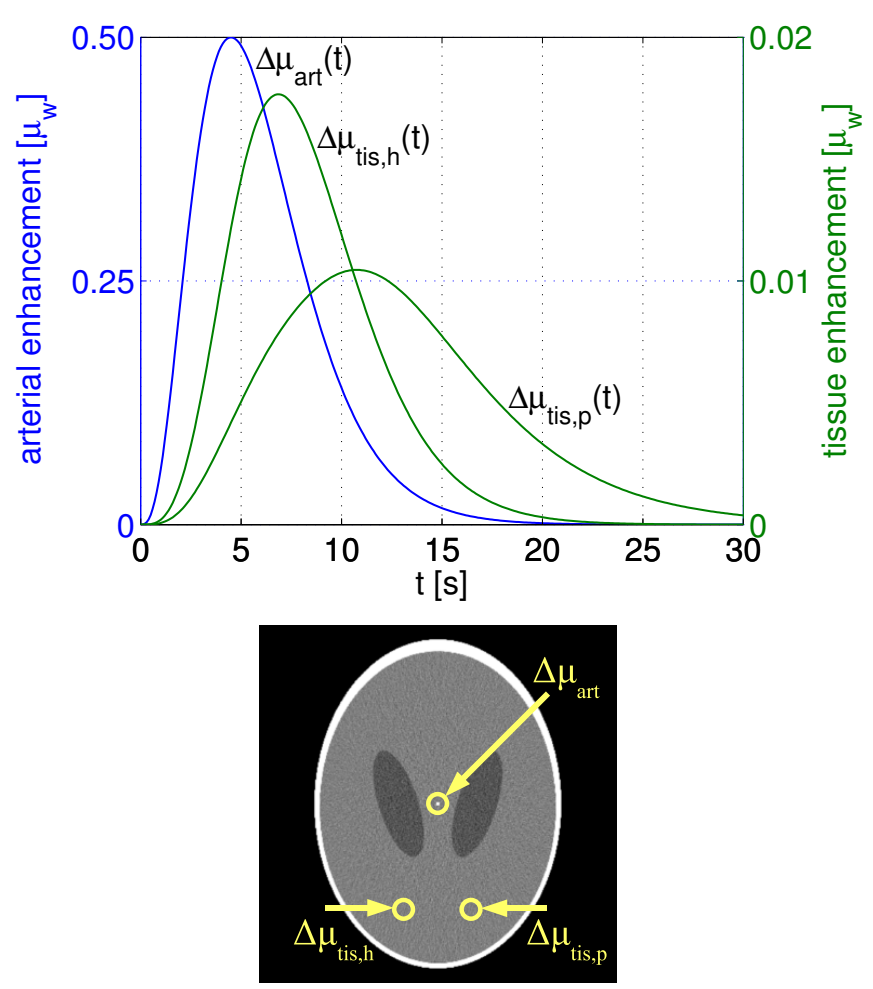

Fig. 4. Top: Synthetic time-attenuation curves, here without noise, corresponding to a large artery $\left(\Delta \mu_{\text {art }}(t)\right.$, left scale), to healthy, normallyperfused tissue $\left(\Delta \mu_{\text {tis, }}(t)\right.$, right scale) and to pathological, hypoperfused tissue $\left(\Delta \mu_{\text {tis,p }}(t)\right.$, right scale) assuming a contrast injection at the aortic arch. The plots show the dynamic enhancement (above the static baseline value) relative to the attenuation $\mu_{\mathrm{w}}$ of water. Note, the arterial peak enhancement is more than 25 times higher than the peak enhancement in tissue. Bottom: Locations of the artery and the tissue regions in the dynamic head phantom.

\section{A. Phantom Description}

We generated two sets of time-attenuation curves representing two different contrast injection methods (intra-venous / aortic arch injection). An intra-venous injection is commonly used in diagnostic perfusion CT imaging. An injection at the aortic arch is feasible in interventional C-arm CT perfusion imaging since patients will be undergoing arterial catheterization for their endovascular therapy [9]. Advantages of the aortic arch injection method are further discussed in Section III-E.

Each set contained three different time-attenuation curves to model the flow of contrast agent through a large artery, a region of normally-perfused tissue and a region of hypoperfused tissue. In each set the arterial enhancement $\Delta \mu_{\text {art }}(t)$ was modeled using the following gamma-variate function [14], [20]:

$$
\Delta \mu_{\mathrm{art}}(t)=\frac{A}{(\alpha \beta \exp (-1))^{\alpha}} \tau^{\alpha} \exp (-\tau / \beta) H(\tau) .
$$

Here, $H(\tau)$ is the unit step function, and $\alpha=3.0$ and $\beta=1.5$ are shape parameters that were also suggested in [20]. The dimensionless quantity $\tau=\left(t-t_{0}\right) / \eta$ depends on the bolus arrival time $t_{0}$ and the time scaling factor $\eta$ which are both measured in s. The factor $\eta$ controls the full width at half maximum of the curve. The initial values were $t_{0}=0$ and $\eta=1$ but, as will be explained in more detail later, we varied these values to obtain several different curves.

The maximum dynamic enhancement $A$ above the static baseline value was chosen differently for each set in order to model different injection methods. In the first set an intravenous bolus injection into the antecubital vein with $A=$ $0.3 \mu_{\mathrm{w}}\left(\mu_{\mathrm{w}}=0.18 \mathrm{~cm}^{-1}\right.$ is the X-ray attenuation of water) was assumed which is typical for diagnostic CT perfusion imaging [21]. In the second set $A=0.5 \mu_{\mathrm{w}}$ was chosen in order to model an injection at the aortic arch as it was carried out in the in vivo studies, see Section IV for details.

In each set the time-attenuation curves $\Delta \mu_{\mathrm{tis}}(t)$ in tissue were computed using the indicator-dilution theory [13], [20],

$$
\begin{aligned}
\Delta \mu_{\mathrm{tis}}(t) & =\rho \mathrm{CBF} \int_{0}^{t} \Delta \mu_{\mathrm{art}}(\tau) r(t-\tau) \mathrm{d} \tau \\
r(t) & = \begin{cases}1, & \text { for } t<T_{0} \\
\exp \left(\frac{-\left(t-T_{0}\right)}{\mathrm{MTT}-T_{0}}\right), & \text { for } t \geq T_{0},\end{cases}
\end{aligned}
$$

with $\rho=1.04 \mathrm{~g} / \mathrm{ml}$. We set $T_{0}=0.632 \times$ MTT as suggested in [22]. According to the central volume theorem [13] MTT can be computed as

$$
\mathrm{MTT}=\mathrm{CBV} / \mathrm{CBF} .
$$

We chose $\mathrm{CBF}=60 \mathrm{ml} / 100 \mathrm{~g} / \mathrm{min}$ and $\mathrm{CBV}=4 \mathrm{ml} / 100 \mathrm{~g}$ to generate a time-attenuation curve $\Delta \mu_{\mathrm{tis}, \mathrm{h}}(t)$ for healthy, normally-perfused tissue and chose $\mathrm{CBF}=20 \mathrm{ml} / 100 \mathrm{~g} / \mathrm{min}$ and $\mathrm{CBV}=4 \mathrm{ml} / 100 \mathrm{~g}$ to generate a time-attenuation curve $\Delta \mu_{\mathrm{tis}, \mathrm{p}}(t)$ for pathological, hypoperfused tissue. A plot of all three time-attenuation curves for the case of the aortic arch injection is shown in Figure 4. Note, the peak enhancement in tissue depends linearly on the arterial peak enhancement $A-$ see (8) and (9) — and for the simulated intra-venous injection it is about $0.011 \mu_{\mathrm{w}}$ (healthy tissue) and $0.006 \mu_{\mathrm{w}}$ (pathological tissue) which are typical values for the peak enhancement in gray matter in diagnostic perfusion CT imaging [21].

For each set of time-attenuation curves a dynamic SheppLogan-type head phantom $\mu_{\text {pha }}(\boldsymbol{x}, t)$ was created that contained 3 circular regions of interest (ROI) with varying attenuation values, see Figure 4. The first ROI (radius $1 \mathrm{~mm}$ ) modeled an artery with attenuation values $\mu_{\mathrm{w}}+\Delta \mu_{\text {art }}(t)$. The second and third ROIs (radius $2 \mathrm{~mm}$ each) modeled tissue regions with attenuation values $\mu_{\mathrm{w}}+\Delta \mu_{\mathrm{tis}, \mathrm{h}}(t)$ and $\mu_{\mathrm{w}}+\Delta \mu_{\mathrm{tis}, \mathrm{p}}(t)$, respectively.

The constant attenuation values of the elliptical skull (outer radii $62 \mathrm{~mm}$ and $92 \mathrm{~mm}$ ) and the brain tissue were $2 \mu_{\mathrm{w}}$ and $\mu_{\mathrm{w}}$, respectively. We have chosen the constant attenuation values of the two inner ellipses to be $0.95 \mu_{\mathrm{w}}$. In order to improve the reproducibility of our results, a more detailed description of this phantom along with relevant source code is available online [23].

\section{B. Investigations}

The following procedures were applied to each phantom configuration (intra-venous / aortic arch injection) individually. We simulated C-arm CT scanning of the 2-D phantom with a linear detector array using the scan parameters from set 1 in 


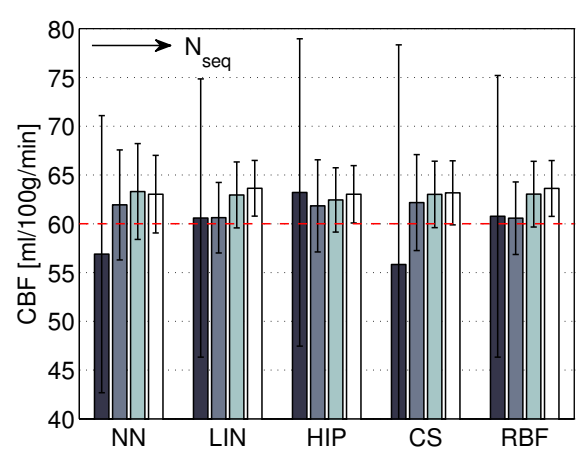

(a) $\mathrm{CBF}$, healthy tissue (GT: $60 \mathrm{ml} / 100 \mathrm{~g} / \mathrm{min})$

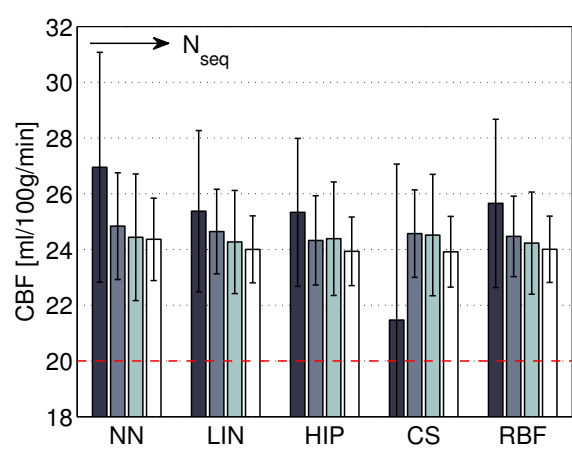

(d) CBF, pathological tissue (GT: $20 \mathrm{ml} / 100 \mathrm{~g} / \mathrm{min})$

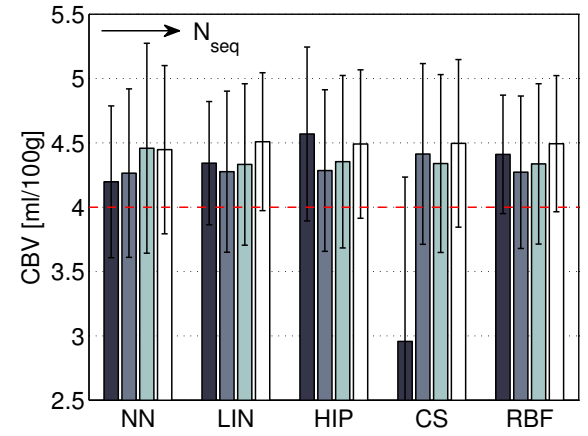

(b) CBV, healthy tissue (GT: $4 \mathrm{ml} / 100 \mathrm{~g})$

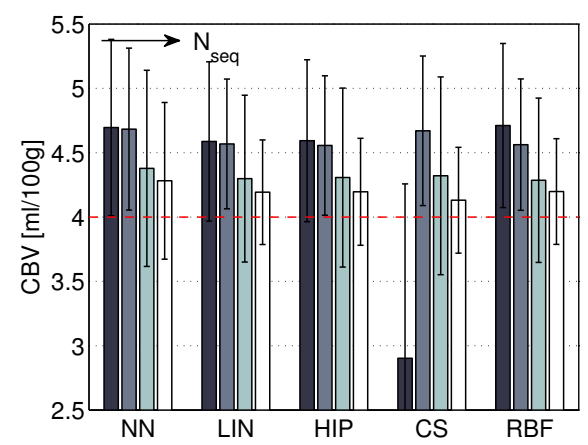

(e) $\mathrm{CBV}$, pathological tissue (GT: $4 \mathrm{ml} / 100 \mathrm{~g})$

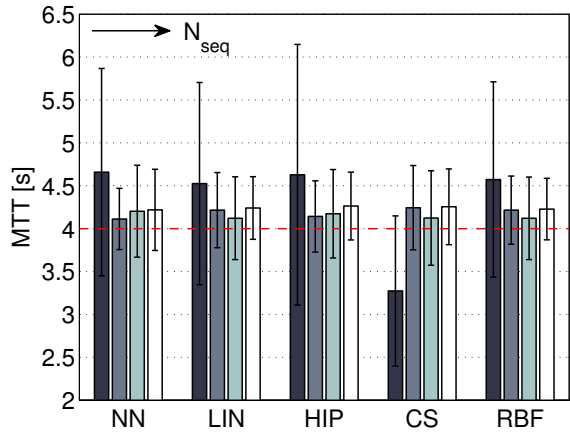

(c) MTT, healthy tissue (GT: 4 s)

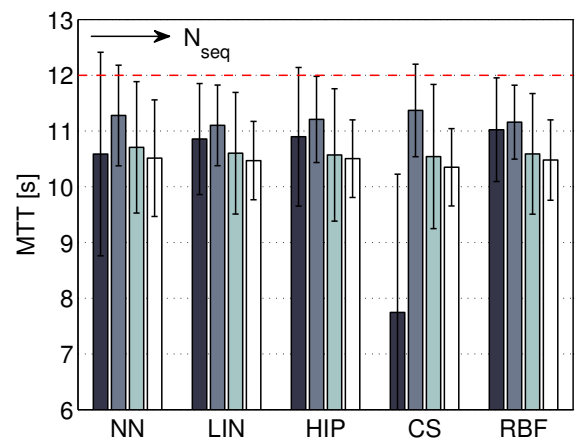

(f) MTT, pathological tissue (GT: $12 \mathrm{~s}$ )

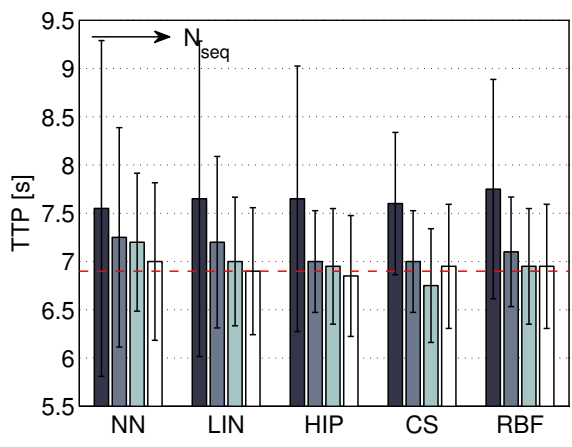

(g) TTP, healthy tissue (GT: $6.9 \mathrm{~s})$

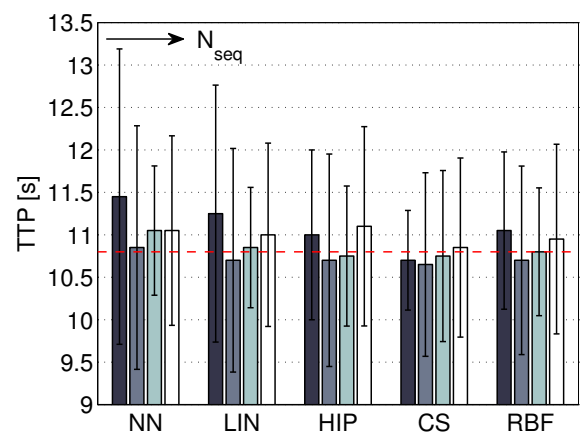

(h) TTP, pathological tissue (GT: $10.8 \mathrm{~s})$

Fig. 5. Simulation with noisy, synthetic data corresponding to $(\mathrm{a}-\mathrm{c}, \mathrm{g})$ healthy and $(\mathrm{d}-\mathrm{f}, \mathrm{h})$ pathological tissue. The graphs show mean and standard deviation of different measures with known ground truth (GT, dashed line) for different interpolation methods (abbreviations explained in Section II-D) and numbers $N_{\text {seq }} \in\{1,2,3,4\}$ of interleaved sequences. The different colors of the bars correspond to values from $N_{\text {seq }}=1$ (dark) to $N_{\text {seq }}=4$ (bright). Note, the CBF, MTT, and TTP plots have different scalings of the ordinate for the cases of healthy and pathological tissue.

Table I. Poisson-distributed noise was added to the projection values of $\mu_{\mathrm{pha}}(\boldsymbol{x}, t)$ assuming an emitted X-ray flux density of $2.1 \times 10^{6}$ photons per $\mathrm{mm}^{2}$ at the source-to-detector distance as in [4].

Different numbers $N_{\text {seq }} \in\{1,2,3,4\}$ of scan sequences and different numbers $M=\{1,2,3,4,5,6,12,18\}$ of angular interpolation intervals were used. We applied the 5 interpolation functions from Section II-D and set the interpolation time step to $0.5 \mathrm{~s}$. These 160 different combinations of IS-PRI parameters were used to simulate scanning of the phantom $\mu_{\mathrm{pha}}(\boldsymbol{x}, t)$. For each instance the scanning was repeated 10 times each time having a different noise realization and different values for $t_{0}$ and $\eta$. These values were uniformly distributed in the intervals $t_{0} \in\left[0,\left(T_{\text {rot }}+T_{\mathrm{w}}\right)[\right.$ and $\eta \in[0.85,1.15]$, respectively. This was done in order to take into account variations due to different, relative shifts between start of the scanning and the bolus arrival and also variations due to different shapes of the curves. The thickness of the reconstructed slices was $9.6 \mathrm{~mm}$ which is a typical value for neuro perfusion CT data. The reconstruction of thick slices was realized by averaging 16 noisy projections, i.e. the slice thickness divided by the detector pixel size, for each view-angle. The in-plane voxel spacing was $0.2 \mathrm{~mm}$.

In order to analyze the reconstructed data we have focused on the following two aspects. First, we investigated the accuracy of measured perfusion values (CBF, CBV, MTT, TTP) for the healthy and pathological tissue. Second, we investigated the reconstruction artifacts due to inconsistent data around the simulated artery, cf. Section II-A2.

The perfusion parameters CBF, CBV and MTT were computed using a standard deconvolution-based perfusion analysis based on the truncated SVD algorithm with a fixed threshold 


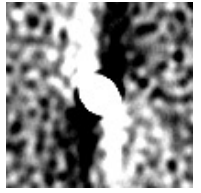

(a) $M=1$

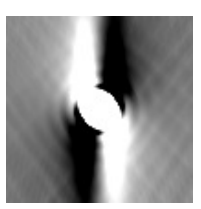

(e) $M=1$

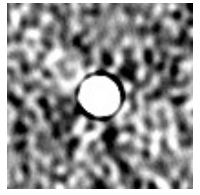

(b) $M=3$

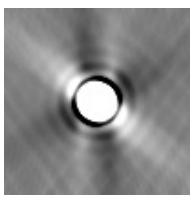

(f) $M=3$

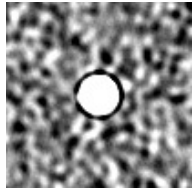

(c) $M=6$

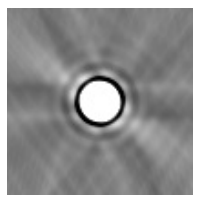

(g) $M=6$

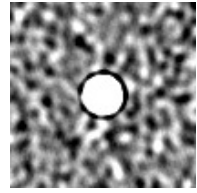

(d) $M=18$

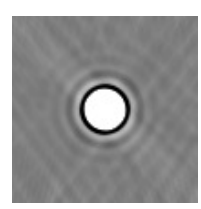

(h) $M=18$
Fig. 8. Reconstruction of a simulated arterial vessel with time-varying attenuation values from $(\mathrm{a}-\mathrm{d})$ noisy and $(\mathrm{e}-\mathrm{h})$ noise-free input data using $N_{\text {seq }}=3$ interleaved sequences. $M$ is the number of angular interpolation intervals. The windowing is from $-10 \mathrm{HU}$ (black) to $+10 \mathrm{HU}$ (white).

value of $20 \%$ of the maximum singular value as described in [13]. In this algorithm the arterial time curve $\mu_{\text {art }}(t)$ and the tissue time curve $\mu_{\mathrm{tis}}$ are deconvolved and the peak value and the integral of this deconvolved function give the $\mathrm{CBF}$ and the $\mathrm{CBV}$, respectively, whereas MTT is the ratio of both, cf. (11). TTP was determined directly from the tissue time curves.

We quantified the reconstruction artifact around the artery by measuring the average absolute deviation from the ground truth value in an annular region around the artery. The following expression defines the pixel positions $\boldsymbol{x}_{i}(i=$ $\left.0, \ldots, N_{\mathrm{i}}-1\right)$ that lie within this annular region:

$$
r_{\text {art }}^{2} \leq x_{i}^{2}+y_{i}^{2} \leq\left(3 r_{\text {art }}\right)^{2} .
$$

We set the outer radius to $3 r_{\text {art }}$ after initial evaluation of different radii in order to have the majority of the artifact within the annulus ring. The measure $\chi_{\text {art }}$ of the mean reconstruction artifact for a given time point $t_{\mathrm{est}}$ is defined as

$$
\chi_{\text {art }}\left(t_{\mathrm{est}}\right)=\frac{1}{N_{\mathrm{i}}} \sum_{i=0}^{N_{\mathrm{i}}-1}\left|\tilde{\mu}_{\mathrm{rec}}\left(\boldsymbol{x}_{i}, t_{\mathrm{est}}\right)-\mu_{\mathrm{pha}}\left(\boldsymbol{x}_{i}, t_{\mathrm{est}}\right)\right| .
$$

\section{Results: Simulated Aortic Arch Injection}

Figure 5 shows the mean and standard deviation of the computed perfusion parameters CBF, CBV, MTT, and TTP from reconstructions with a constant number $M=6$ of angular interpolation intervals and different interpolation methods. Results with different numbers $M$ will be discussed later. According to the central volume theorem, see (11), only two of the three parameters CBF, CBV and MTT are independent; however, we present the results of all of these parameters for a more comprehensive evaluation.

Generally, the standard deviation of the computed parameter values, which is a measure for the variability, decreases when the number $N_{\text {seq }}$ of interleaved sequences increases. Especially the standard deviation of CBF, MTT, and TTP (healthy tissue) decreases significantly when $N_{\text {seq }}$ is increased from 1 to 2. For example, with linear interpolation the standard deviation of CBF in healthy tissue decreases from $14.3 \mathrm{ml} / 100 \mathrm{~g} / \mathrm{min}$ to $3.6 \mathrm{ml} / 100 \mathrm{~g} / \mathrm{min}$ when two interleaved sequences instead of one sequence are used. For pathological tissue these values are $2.9 \mathrm{ml} / 100 \mathrm{~g} / \mathrm{min}$ and $1.5 \mathrm{ml} / 100 \mathrm{~g} / \mathrm{min}$.

The mean TTP values get closer to the ground truth value for increasing $N_{\text {seq }}$ and the mean CBF and mean CBV values tend to get overestimated, especially for pathological tissue. The mean MTT values show different trends for increasing $N_{\text {seq }}$ for the cases of healthy (they are closer to the ground truth) and pathological tissue (they tend to get underestimated).

Figure 6 shows the CBF, CBV, MTT, and TTP of the simulated pathological tissue as a function of different number $M$ of interpolation intervals when using linear interpolation. For $N_{\text {seq }} \in\{3,4\}$ the mean of the estimations of CBV, MTT, and TTP varies with increasing $M$ values while the $\mathrm{CBF}$ estimation is nearly unaffected by different $M$ values. It can be observed that the results with $M \in\{12,18\}$ are similar to the results with $M=6$. Generally, the estimation of the perfusion parameters does not improve significantly when increasing $M$ for neither the pathological tissue (Figure 6) nor for the healthy tissue (data omitted for brevity).

Results for the reconstruction artifact around the simulated artery are shown in Figure 7. Figure 7(c) shows $\chi_{\text {art }}(t)$ for different time points $t$ when using the linear interpolation method. If $N_{\text {seq }}>1$ then the value of $\chi_{\text {art }}(t)$ decreases for $M=6$ when compared to $M=1$. For comparison of different interpolation methods, in Figure 7(a)-(b) the mean and standard deviation is plotted for a reconstruction time point during the arterial outflow phase $\left(t_{\text {est }}=9 \mathrm{~s}\right.$, see Figure 4$)$. The value of $\chi_{\text {art }}(9 \mathrm{~s})$ from a reconstruction without any simulated contrast flow is indicated with a dashed line, this value is purely due to the noise in the projection images and thus also the reconstructed images, cf. definition of $\chi_{\text {art }}$ in (13).

As a visual example Figure 8 shows reconstructions of the simulated arterial vessel at the time point $t_{\text {est }}=9 \mathrm{~s}$ for different numbers $M$, here with an in-plane voxel spacing of $0.1 \mathrm{~mm}$. Additionally, also the results obtained from noise-free input data are presented in this figure.

\section{Results: Simulated Intra-venous Injection}

The results based on the simulated intra-venous injection show similar trends - when changing $N_{\text {seq }}$, for example - as the results corresponding to the simulated aortic arch injection (Section III-C). However, when comparing the two injection methods the intra-venous injection provides worse results than the aortic arch injection.

For brevity we only show the results of the intra-venous injection for one relevant case. With linear interpolation the standard deviation of $\mathrm{CBF}$ in healthy tissue decreases from $15.0 \mathrm{ml} / 100 \mathrm{~g} / \mathrm{min}$ to $12.1 \mathrm{ml} / 100 \mathrm{~g} / \mathrm{min}$ when two interleaved sequences instead of one sequence are used. For pathological tissue these values are $3.7 \mathrm{ml} / 100 \mathrm{~g} / \mathrm{min}$ and $3.1 \mathrm{ml} / 100 \mathrm{~g} / \mathrm{min}$.

\section{E. Discussion}

The results indicate that when using an aortic arch injection the perfusion parameters can be estimated more reliably when compared to an intra-venous injection. This can be attributed 


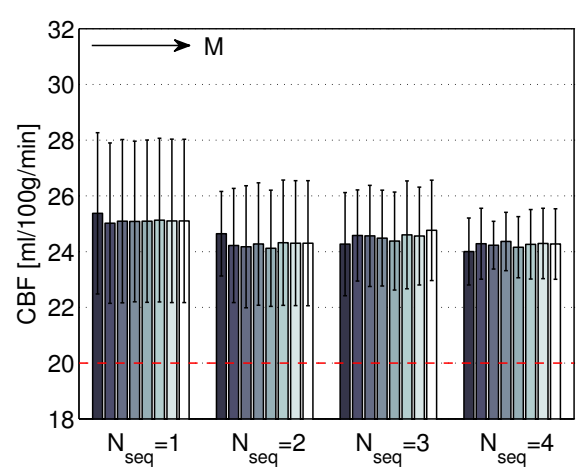

(a) cerebral blood flow (GT: $20 \mathrm{ml} / 100 \mathrm{~g} / \mathrm{min})$

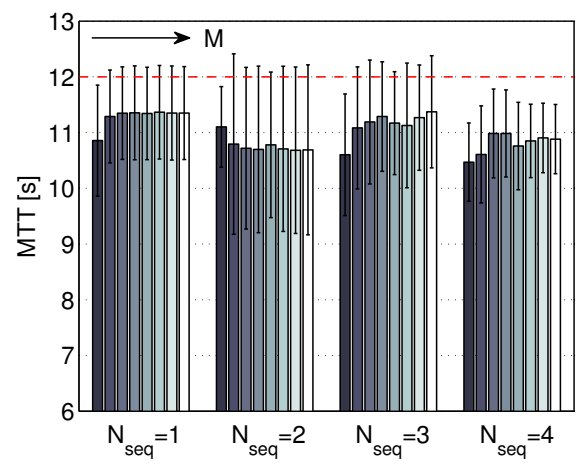

(c) mean transit time (GT: $12 \mathrm{~s})$

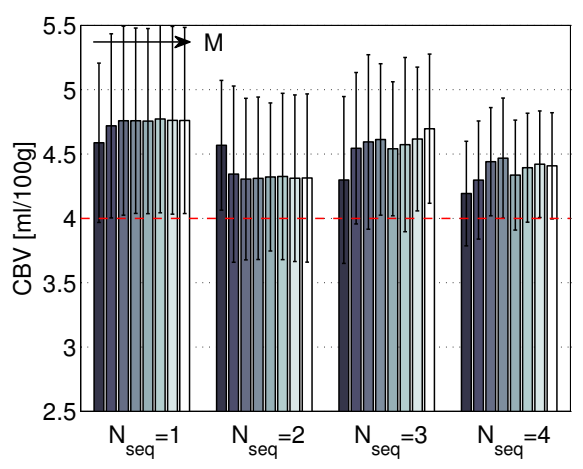

(b) cerebral blood volume (GT: $12 \mathrm{ml} / 100 \mathrm{~g})$

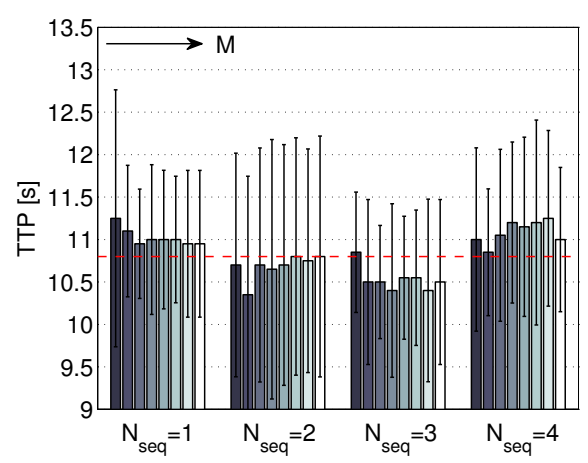

(d) time-to-peak (GT: $10.8 \mathrm{~s})$

Fig. 6. Simulation with noisy, synthetic data: Mean and standard deviation of estimated CBF, CBV, MTT, and TTP with known ground truth (GT, dashed line) depending on different number $N_{\text {seq }}$ of interleaved sequences and different numbers $M \in\{1,2,3,4,5,6,12,18\}$ of angular interpolation intervals (the bars are ordered in increasing numbers of $M$ ) investigated using pathological tissue and applying linear interpolation as interpolation method.

to the higher peak enhancement in the artery and the tissue regions and thus an improved contrast-to-noise ratio of the reconstructed C-arm CT volumes.

There are also further advantages of the aortic arch injection method. The interleaved scanning with multiple contrast bolus injections is based on the assumption that similar contrast flow patterns can be generated in the patient's brain for each bolus injection. With contrast bolus injections at the aortic arch the bolus travel time to the brain is very short which can improve the reproducibility of the contrast flow pattern.

Furthermore, the fraction of contrast that actually reaches the brain is increased compared to an intra-venous contrast bolus injection. Thus, the necessary total amount of contrast can be reduced. However, a potential disadvantage of the aortic arch injection may be unsymmetrical filling of the arteries with contrast. A symmetrical filling is necessary for comparison of perfusion between the cerebral hemispheres. With an optimized catheter position a symmetrical filling may be achieved but further evaluations are necessary [24].

When using interleaved scanning for perfusion C-arm CT imaging the aortic arch injection method has distinct advantages and we have also used this method in our in vivo studies. In our following discussion we therefore focus on the results obtained with the aortic arch injection method.

The results show that perfusion parameters can be measured with less variability, i.e. lower standard deviation, when several $\left(N_{\text {seq }}>1\right)$ interleaved sequences are used. A few exceptions exist that could be explained due to the specific sampling pattern, see (1), and the shape of the synthetic curves which could be optimal for specific values of $N_{\text {seq }}$. The mean value estimation of the perfusion parameters is not generally improved with an increased number $N_{\text {seq }}$ of interleaved sequences.

In brain perfusion imaging the relative comparison of perfusion values in the healthy and diseased hemispheres is more significant than absolute perfusion values [1]. Therefore, the variability of the measurements is a more important aspect than the mean values.

The relatively higher overestimation of the CBF mean values for the pathological tissue when compared to the healthy tissue can be attributed to the properties of the perfusion image analysis algorithm. We applied the truncated SVD algorithm that is frequently used for this purpose and is also implemented in different perfusion analysis software packages [25]. However, this algorithm is known to underestimate higher CBF values [20], [26] which explains the decrease in overestimation for the higher $\mathrm{CBF}$ values seen in healthy tissue. The additional CBF value offset (for pathological tissue it is typically $4 \mathrm{ml} / 100 \mathrm{~g} / \mathrm{min}$ ) can be attributed to the image acquisition and reconstruction scheme. The arterial input function is characterized by fast contrast agent dynamics and it may be underestimated. Since it is used to normalized the perfusion data the CBF values are overestimated [13].

A reliable differentiation of pathological and healthy tissue is possible based on the results of the computed perfusion values (Figure 5). When using $N_{\text {seq }}=2$ and linear interpo- 


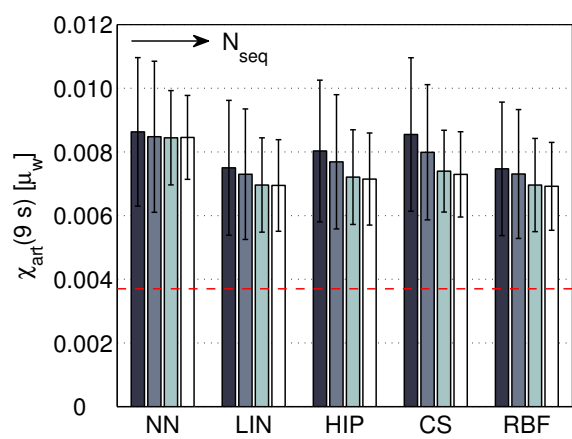

(a) $M=1, N_{\text {seq }}=3$, fixed time: $t=9 \mathrm{~s}$

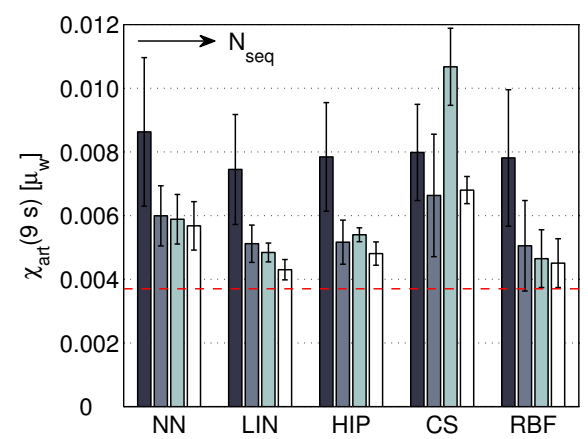

(b) $M=6, N_{\text {seq }}=3$, fixed time: $t=9 \mathrm{~s}$

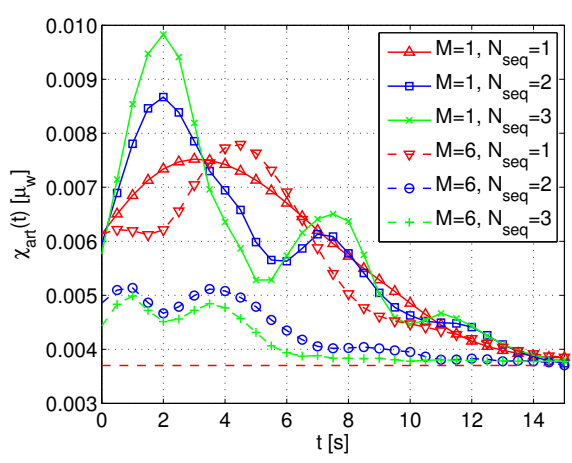

(c) $\chi_{\operatorname{art}}(t)$ as a function of time, LIN interpolation

Fig. 7. Simulation with noisy, synthetic data: (a-b) Mean and standard deviation of the reconstruction artifact $\chi_{\text {art }}(t)$ around the simulated arterial vessel at time point $t=9$ s for different numbers $N_{\text {seq }} \in\{1,2,3,4\}$ of interleaved sequences (the bars are ordered in increasing numbers of $N_{\text {seq }}$ ) and interpolation methods (abbreviations explained in Section II-D). $\mu_{\mathrm{w}}$ is the X-ray attenuation of water. (c) Plot of $\chi_{\text {art }}(t)$ computed with linear interpolation (LIN) as a function of time. The dashed lines indicate the value for the component of $\chi_{\text {art }}$ that is purely due to noise. Optimal results should be close to the value of this dashed line.

lation, for example, the computed CBF range - here defined as mean value \pm 1.96 times the standard deviation - is $53.5-$ $67.7 \mathrm{ml} / 100 \mathrm{~g} / \mathrm{min}$ for healthy and $21.7-27.6 \mathrm{ml} / 100 \mathrm{~g} / \mathrm{min}$ for pathological tissue.

For $N_{\mathrm{seq}} \geq 2$ the performance of the different interpolation methods is comparable. Linear and nearest neighbor interpolation may be favored in practice because they can be implemented such that they are computationally very fast. Of these two methods, linear interpolation shows slightly better results (e.g., lower variability of CBF, CBV, MTT, and TTP measurements for $N_{\mathrm{seq}}=2$ ). With two interleaved sequences the variability for the measured perfusion parameters may already be low enough for application in brain perfusion imaging.

For $N_{\text {seq }}>1$, the variability of the parameters CBF, CBV, and MTT does not decrease for increasing $M$. This effect can be explained by the deconvolution-based perfusion analysis. If $M=1$ then the reconstructed time curves have been low-pass filtered before sampling and interpolation. The lowpass filtering can be approximated by a convolution with a rectangular function of temporal width $T_{\text {rot }}$, see Section II-A2. Note, both the arterial and the tissue curves are low-pass filtered with a similar filter kernel. Mathematically, the deconvolution operation of two functions is invariant to a preceding convolution of each function with the same filter kernel.

We note that other measures of the tissue time curves which do not depend on the normalization with the arterial time curve, such as peak value, can improve for increasing $M$ as has been shown in [8]. However, the investigation of clinically relevant parameters such as $\mathrm{CBF}$ is more significant.

The major advantage of using $M>1$ can be seen in Figures 7 and 8 . When using the PRI approach it is possible to approximate a consistent data set of projection values and to reduce the reconstruction artifacts due to data inconsistencies. It can be seen that for $t=9 \mathrm{~s}$, for example, the artifact is reduced almost completely if $N_{\text {seq }} \geq 2$. This can be explained by the linear slope of the arterial time-attenuation curve at this time point which enables accurate estimation of the unknown data by means of linear interpolation. Choosing higher numbers $M$ of angular interpolation intervals than $M=6$ does not significantly improve the results, although small changes can be seen in the noise-free data of Figure 8. Therefore, we suggest setting $M$ to 6 in order to reduce the computational complexity.

\section{IN Vivo StUdY}

In order to show the clinical feasibility of our novel IS-PRI approach and to validate it under realistic conditions we have conducted an in vivo brain perfusion study with 5 healthy pigs where we used perfusion CT as reference for the validation.

\section{A. Material and Methods}

The following procedure was performed under institutional review board approval for each of the 5 perfusion-normal pigs (mean weight $54 \pm 4.7 \mathrm{~kg}$ ). The pig was sedated and placed on a respirator. A 5-French diffusion catheter (Vanguard, Medrad Inc, Idianola, PA, USA) was placed at the root of the aortic arch under fluoroscopic guidance. During the study we injected iodinated contrast agent (Omnipaque, $350 \mathrm{mg}$ iodine $/ \mathrm{ml}$, Nycomed, Princeton, NJ, USA) diluted with an equal amount of saline using a programmable dual head power injector (Accutron HP-D, Medtron AG, Saarbrücken, Germany). Using this injector, delay times could be programmed with $0.1 \mathrm{~s}$ precision. We injected each bolus at a rate of $6 \mathrm{ml} / \mathrm{s}$ for $8 \mathrm{~s}$, resulting in $24 \mathrm{ml}$ of pure contrast agent per injection.

We acquired $N_{\mathrm{seq}}=6$ interleaved sequences with a clinical C-arm CT system (Axiom Artis $d$ TA with syngo DynaCT, Siemens AG, Healthcare Sector, Forchheim, Germany) using the C-arm CT scan parameters from set 2 of Table I. Retrospectively, we created 3 additional subsets of sequences with $N_{\text {seq }}=1\left(1^{\text {st }}\right.$ sequence of the superset $), N_{\text {seq }}=2\left(1^{\text {st }}\right.$ and $4^{\text {th }}$ sequence of the superset $)$ and $N_{\text {seq }}=3\left(1^{\text {st }}, 3^{\text {rd }}\right.$ and $5^{\text {th }}$ sequence of the superset). The size of each reconstructed volume was $256 \times 256 \times 256$ isotropic voxels with a voxel side length of $0.5 \mathrm{~mm}$ and the interpolation sampling interval was $1 \mathrm{~s}$. Based on our experience from the simulations we set the number $M$ of interpolation intervals to 6 and we used the linear interpolation method. 
For validation we acquired a perfusion CT (first 2 animals: Somatom Sensation 64, Siemens AG, Healthcare Sector, Erlangen, Germany; last 3 animals: LightSpeed 16, GE Healthcare, Milwaukee, WI, USA) for each of the 5 animals with the same injection parameters as for the C-arm CT scans. Baseline images were acquired and subtracted from the following images to measure the enhancement due to contrast flow. The measured enhancement is used for the data analysis and it is actually independent of residual contrast that may originate from the previous perfusion $\mathrm{C}$-arm CT exam. The data from the perfusion $\mathrm{CT}$ exam was reconstructed with a pixel size of $0.39 \times 0.39 \mathrm{~mm}^{2}$, a slice thickness of $9.6-$ $10.0 \mathrm{~mm}$ and a temporal sampling interval of 0.5-1.0 s. For the $\mathrm{C}$-arm CT data we created reconstructions with similar slice thicknesses by applying a moving average filter (kernel size $9.5-10.0 \mathrm{~mm}$ ) perpendicular to the orientation of the reconstructed slices.

After reconstruction of the 4-D C-arm CT data set we evaluated qualitatively the time-attenuation curves in an arterial and a venous vessel and in a tissue region. We carried out a quantitative evaluation using the computed perfusion parameter maps.

For this evaluation we first registered the 3-D C-arm CT perfusion maps onto the 2-D perfusion maps (2-3 slices per animal) obtained with CT. We have used a rectangular grid with a line spacing of 10 pixels to subdivide the whole brain area into square ROIs. For each ROI the mean perfusion values (CBF, CBV, MTT) were computed using our in-house software based on the truncated SVD algorithm [13].

The linear correlation $r$ between the perfusion CT map and the (registered) perfusion C-arm CT map was determined by using the data from all ROIs. In order to decrease the influence of large vessels we additionally applied a vascular pixel elimination (VPE) similar to the method described in [27]. If the mean CBV value from perfusion CT in a certain square ROI was above the threshold value of $8 \mathrm{ml} / 100 \mathrm{~g}$ (this value was suggested in [27] for human data and we assumed it also applies to the data from our in vivo pig studies because of the similarities of the human brain and the pig brain [28], [29]) then the data from this ROI was not used for the correlation analysis.

An alternative evaluation method would be to manually select ROIs in different gray and white matter regions as done in [9]. The evaluation conducted in this work does not require manual selection of ROIs and is therefore user-independent.

\section{B. Results}

Figure 9 shows the locations of the arterial and venous vessels and the tissue regions that were selected for investigation of the time-attenuation curves. The time-attenuation curves from one animal are shown in Figure 10. With increasing $N_{\text {seq }}$ the full width at half maximum of the reconstructed curves decreases and gets closer to the value from the reference CT. The curves obtained with IS-PRI are generally smoother than the curves from CT. The smoothest curves are obtained with $N_{\text {seq }}=1$. These qualitative findings also hold for the results of the other 4 animals of our study.

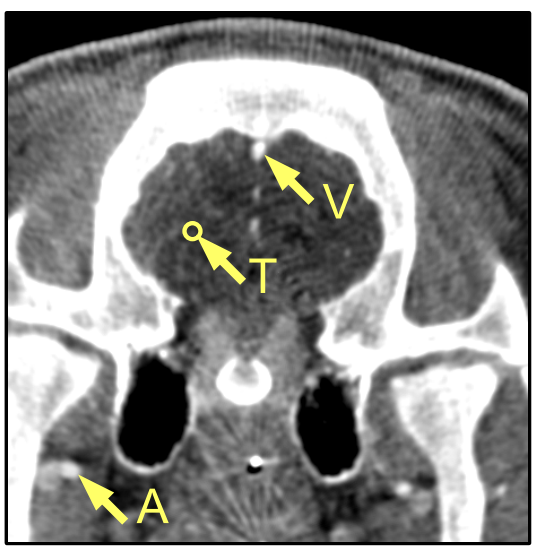

Fig. 9. Coronal C-arm CT image of a pig head that shows the locations of the extra-cranial arterial (A) and intra-cranial venous (V) vessels and the tissue $(\mathrm{T})$ region that were selected for plotting of the time-attenuation curves shown in Figure 10. The windowing range is from $-75 \mathrm{HU}$ to $+375 \mathrm{HU}$.

A representative 3-D CBF data set of one animal is given in Figure 11 by displaying 3 orthogonal planes through the volume. No severe asymmetry can be seen in the transversal and coronal planes as would be expected from a healthy animal. Figure 12 shows a comparison of the CBF maps of one animal obtained with perfusion $\mathrm{CT}$ and with perfusion C-arm CT using IS-PRI $\left(N_{\text {seq }}=2, M=6\right)$.

Table II shows the linear correlation coefficients $r$ between perfusion CT and perfusion C-arm CT parameters for the results with and without VPE. For brevity, we have omitted the detailed results for MTT. The mean correlations for MTT were generally lower ( $r=0.12$ with VPE, $r=0.35$ without VPE for $N_{\text {seq }}=2$ ) but also showed an increase for increasing $N_{\text {seq }}$ $\left(r=0.31\right.$ with VPE, $r=0.51$ without VPE for $N_{\text {seq }}=3$ ).

\section{Discussion}

The results from Figure 10 show that the time-attenuation curves measured with the IS-PRI approach are comparable to those measured with CT. The smoothness of the curves with IS-PRI could be explained by the potential undersampling, especially at low numbers $N_{\text {seq }}$, and the interpolation, both of which act as a low-pass filtering.

Compared to conventional multi-slice perfusion CT it is possible to measure volumetric perfusion with our IS-PRI approach, cf. Figure 11. A detailed discussion of the advantages of volumetric perfusion measurement for stroke diagnosis is provided in [30].

The mean correlation of the computed C-arm CT perfusion values with perfusion CT increases for increasing $N_{\text {seq }}$ regardless of whether vascular pixels are kept or eliminated. While the mean correlation is highest when vascular pixels are included in the analysis, it is also very high if the vascular pixels are removed. ${ }^{1}$ In that case the improvement when using

\footnotetext{
${ }^{1}$ It has been observed that when removing the vascular pixels from the statistical analysis the decrease of the covariance between the measured CT and C-arm CT values is relatively stronger than the decrease of the product of their standard deviations. This explains the decrease of the linear correlation coefficient - which is the ratio of these two quantities - when VPE is applied.
} 


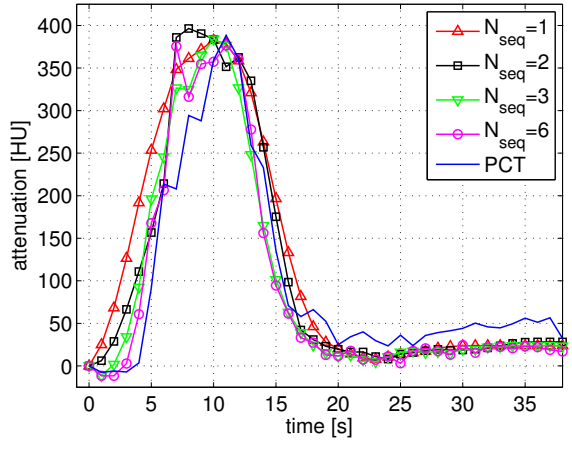

(a) artery

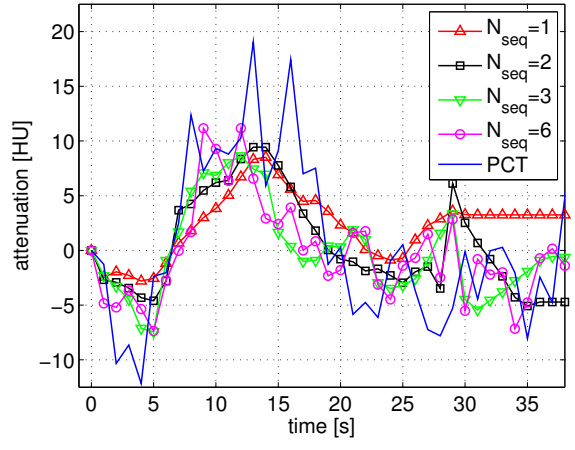

(b) tissue

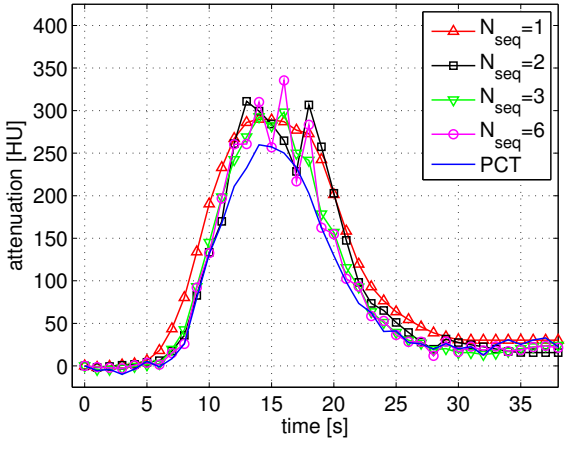

(c) vein

Fig. 10. Reconstructed time-attenuation curves obtained from one pig in our in vivo studies using different numbers $N_{\text {seq }} \in\{1,2,3,4\}$ of interleaved sequences and $M=6$ angular interpolation intervals. The data from a perfusion CT (PCT) exam is given as reference. For each curve the first sample point value was subtracted to allow for better comparison.

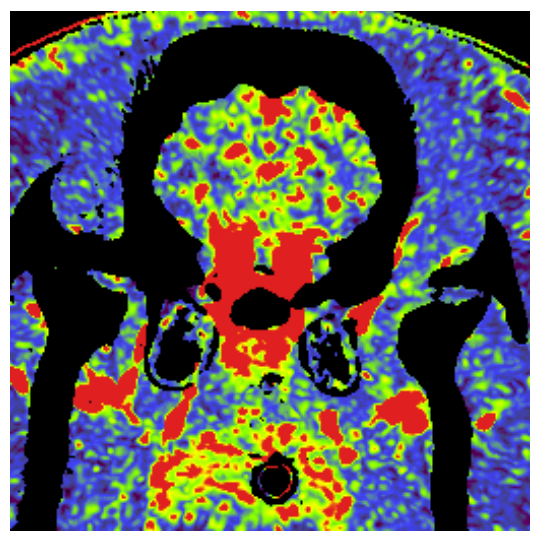

(a) coronal

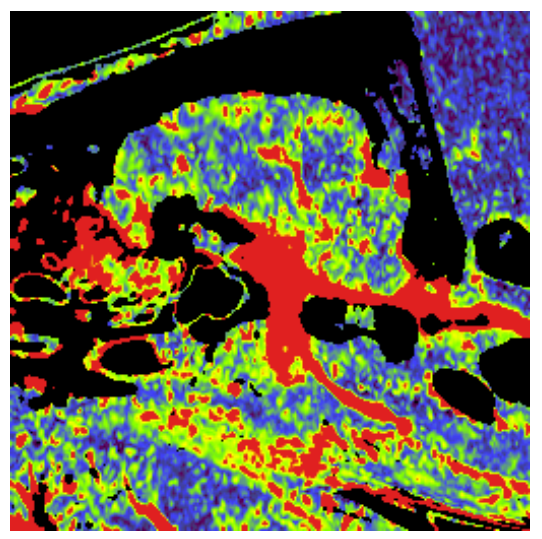

(b) sagittal

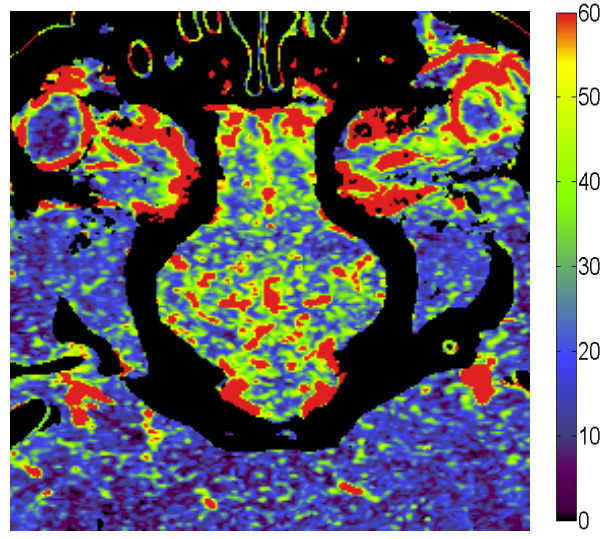

(c) transversal

Fig. 11. 3-D CBF data (unit: $\mathrm{ml} / 100 \mathrm{~g} / \mathrm{min}$ ) of a perfusion-normal pig computed from 4-D data acquired using the IS-PRI approach. The color bar applies to all three images.

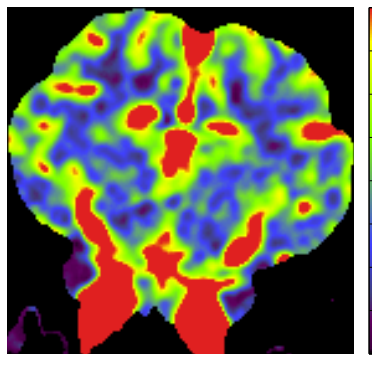

(a) PCT, slice 1

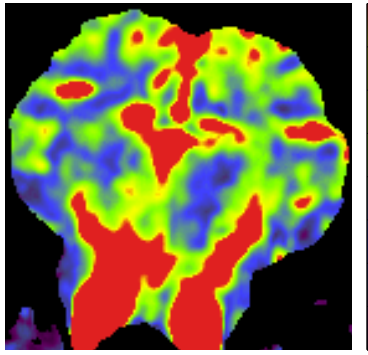

(d) IS-PRI, slice 1

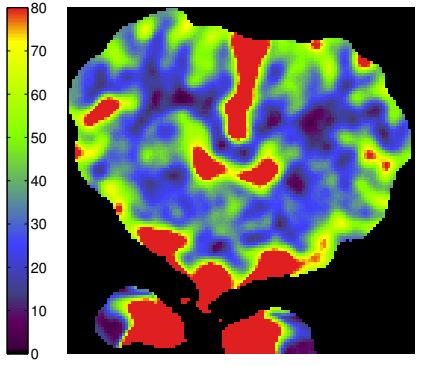

(b) PCT, slice 2

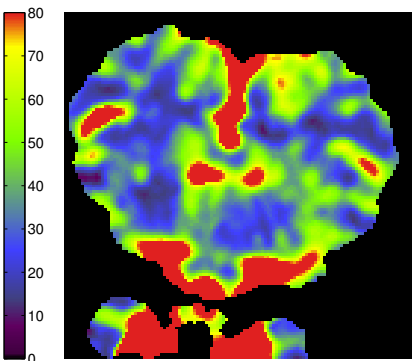

(e) IS-PRI, slice 2

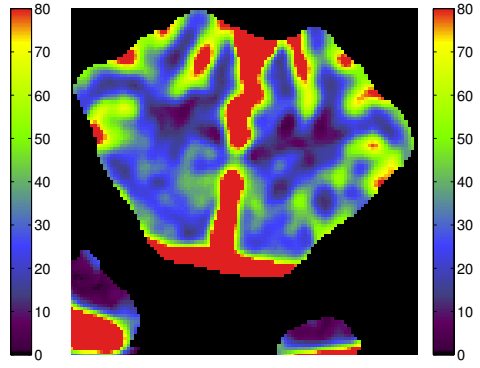

(c) PCT, slice 3

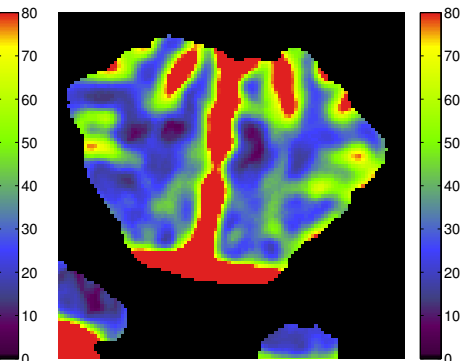

(f) IS-PRI, slice 3

Fig. 12. CBF maps (units: $\mathrm{ml} / 100 \mathrm{~g} / \mathrm{min}$ ) of a perfusion-normal pig obtained with (a-c) perfusion CT (PCT) and (d-f) perfusion C-arm CT using the IS-PRI approach. The 3-D volume CBF data from IS-PRI was registered onto the 2-D maps from perfusion CT. 
TABLE II

CORRELATION $r$ BETWEEN PERFUSION PARAMETERS THAT WERE MEASURED WITH CT AND C-ARM CT IN 5 HEALTHY PIGS (LABELED FROM A TO E). VPE: VASCULAR PIXEL ELIMINATION, SD: STANDARD DEVIATION.

\begin{tabular}{lllllll|l}
\hline & $N_{\text {seq }}$ & A & B & C & D & E & mean \pm SD \\
\hline $\mathrm{CBF}[\mathrm{ml} / 100 \mathrm{~g} / \mathrm{min}]$, w/o VPE & 1 & 0.97 & 0.92 & 0.91 & 0.87 & 0.95 & $0.92 \pm 0.04$ \\
$\mathrm{CBF}[\mathrm{ml} / 100 \mathrm{~g} / \mathrm{min}]$, w/ VPE & 1 & 0.79 & 0.80 & 0.58 & 0.54 & 0.62 & $0.67 \pm 0.12$ \\
$\mathrm{CBV}[\mathrm{ml} / 100 \mathrm{~g}]$, w/o VPE & 1 & 0.98 & 0.96 & 0.92 & 0.87 & 0.96 & $0.94 \pm 0.05$ \\
$\mathrm{CBV}[\mathrm{ml} / 100 \mathrm{~g}]$, w/ VPE & 1 & 0.75 & 0.73 & 0.52 & 0.42 & 0.49 & $0.58 \pm 0.15$ \\
\hline $\mathrm{CBF}[\mathrm{ml} / 100 \mathrm{~g} / \mathrm{min}]$, w/o VPE & 2 & 0.97 & 0.95 & 0.93 & 0.88 & 0.94 & $0.94 \pm 0.03$ \\
$\mathrm{CBF}[\mathrm{ml} / 100 \mathrm{~g} / \mathrm{min}]$, w/ VPE & 2 & 0.79 & 0.82 & 0.74 & 0.55 & 0.63 & $0.71 \pm 0.11$ \\
$\mathrm{CBV}[\mathrm{ml} / 100 \mathrm{~g}]$, w/o VPE & 2 & 0.98 & 0.96 & 0.93 & 0.89 & 0.96 & $0.94 \pm 0.04$ \\
$\mathrm{CBV}[\mathrm{ml} / 100 \mathrm{~g}]$, w/ VPE & 2 & 0.78 & 0.77 & 0.59 & 0.46 & 0.53 & $0.63 \pm 0.14$ \\
\hline $\mathrm{CBF}[\mathrm{ml} / 100 \mathrm{~g} / \mathrm{min}]$, w/o VPE & 3 & 0.93 & 0.95 & 0.94 & 0.89 & 0.93 & $0.93 \pm 0.02$ \\
$\mathrm{CBF}[\mathrm{ml} / 100 \mathrm{~g} / \mathrm{min}]$, w/ VPE & 3 & 0.80 & 0.83 & 0.78 & 0.59 & 0.65 & $0.73 \pm 0.10$ \\
$\mathrm{CBV} \mathrm{[ml/100g],} \mathrm{w/o} \mathrm{VPE}$ & 3 & 0.98 & 0.96 & 0.94 & 0.91 & 0.95 & $0.95 \pm 0.03$ \\
$\mathrm{CBV} \mathrm{[ml/100g],} \mathrm{w/} \mathrm{VPE}$ & 3 & 0.77 & 0.76 & 0.71 & 0.51 & 0.61 & $0.67 \pm 0.11$ \\
\hline
\end{tabular}

increasing numbers $N_{\text {seq }}$ is most prominent. The standard deviation of the mean correlation decreases for increasing $N_{\text {seq. }}$. The evaluation with VPE may be clinically more significant, in order to evaluate tissue perfusion, than the evaluation without VPE. Nevertheless, the results without VPE show the correlation between perfusion CT and perfusion C-arm CT values on a broader range of values.

We did not evaluate higher numbers $M$ of angular interpolation intervals as the results from the simulations did not show a significant improvement for $M>6$. The simulations showed that reconstruction artifacts could be reduced with $M=6$ compared to $M=1$. With in vivo data these reconstruction artifacts were difficult to evaluate due to the lack of ground truth data.

In order to increase the contrast-to-noise ratio (CNR) of the measured tissue time-attenuation curves and to increase the reproducibility of the contrast flow patterns we used a contrast bolus injection at the aortic arch, cf. Section III-E. Intra-venous contrast injection methods may be investigated in future animal studies. Note, ECG-triggered contrast bolus injections, which were not performed in this study due to hardware constraints, could reduce the influence of the cardiac cycle [15] which could further increase the reproducibility of the contrast flow patterns.

Our study was not optimized for X-ray dose reduction. When used in clinical practice the applied dose of a protocol with two interleaved scan sequences may be similar to the applied dose of current volume perfusion CT protocols [31].

A more detailed analysis of the clinical data focusing on clinical aspects such as different injection protocols and a differentiation of gray and white matter was carried out in [9] where further statistical measures (Bland-Altman plots, concordance correlation coefficients) were also investigated. In [9] the correlation between perfusion CT and perfusion $\mathrm{C}$-arm CT was computed using manually selected circular ROIs and a linear correlation of $r=0.88$ was obtained with two interleaved sequences. Also with the automatic, userindependent ROI selection approach as carried out in this work the correlation of $\mathrm{CBF}$ and $\mathrm{CBV}$ is very high (ranging from $r=0.63$ to $r=0.94$ for $N_{\text {seq }}=2$ ).
Our studies were based on perfusion-normal pigs. Note, there is no minimally invasive stroke model for pigs since the rete mirabile does not permit endovascular access to the anterior cerebrovasculature [29]. Further studies involving animal stroke models or human stroke patients are necessary for the validation of our methods and will be carried out in the future.

\section{Outlook on Future DeVelopments}

The IS-PRI approach presented in this paper enables, for the first time, perfusion imaging using $\mathrm{C}$-arm $\mathrm{CT}$ in the interventional suite. Future research in this field may focus on further increasing the accuracy of the technique, and to reduce the amount of contrast agent and X-ray radiation that is applied to the patient. These goals could be accomplished by new image reconstruction algorithms or new C-arm CT system technologies. In this section, we will give an outlook on potential future developments.

\section{A. Dual-source Bi-plane System}

As discussed in Section III-E, two interleaved sequences may already provide results that can be used for a reliable stroke diagnosis. However, two separate injections are required which increase the contrast agent dose and lead to a longer examination time. The necessity for two injections could be relaxed if a bi-plane $\mathrm{C}$-arm angiography system was used where both planes could acquire projections simultaneously during the $\mathrm{C}$-arm rotation. In that case, two interleaved-type sequences could be acquired with only one contrast bolus injection.

Figure 13 shows how the PRI approach could be applied to data acquired with a bi-plane system. The two planes of the bi-plane system are mounted at an angle of $\Lambda / 2$ in relation to each other. With current bi-plane systems it is not possible to perform simultaneous data acquisition using both planes. However, if simultaneous acquisition was feasible, and if a biplane system was actually available in the interventional suite, the aforementioned modified PRI approach could be used as an alternative to the dual bolus approach. 


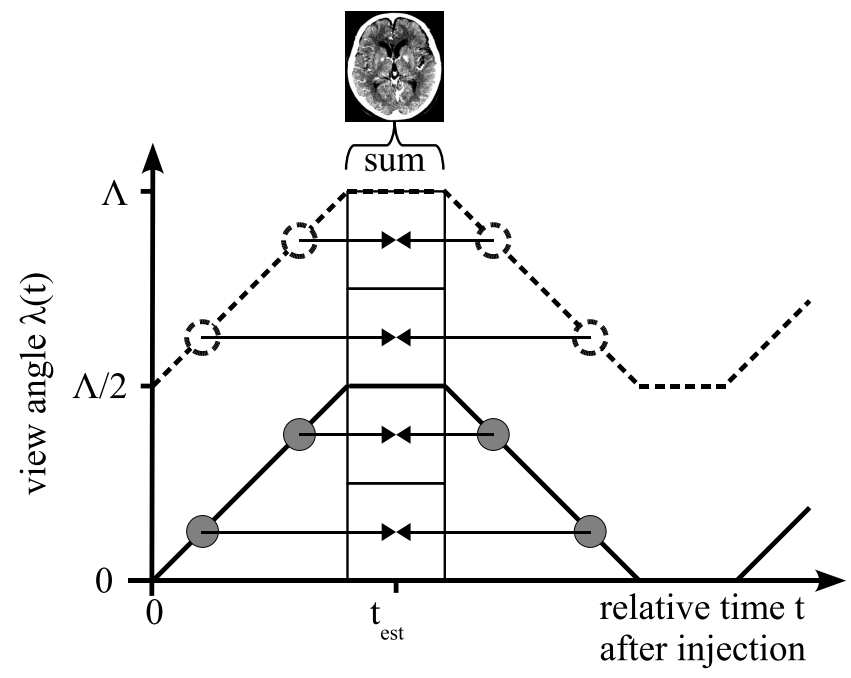

Fig. 13. Partial reconstruction interpolation applied to data acquired using a bi-plane system. See Figure 3(b) for a comparison with the case for a monoplane system.

\section{B. Constrained Image Reconstruction Techniques}

In this section, we will discuss how different constrained image reconstruction techniques could further enhance the ISPRI approach presented in this paper or even lead to new kinds of approaches.

It was recently shown that 4-D vascular blood flow information can be obtained from projection data acquired during a slow C-arm rotation using a C-arm CT system [32], [33]. This novel technique uses a limited number of projections and a constrained image reconstruction for obtaining reconstructed volumes corresponding to different time frames at short intervals. The vascular blood flow information alone cannot be used to quantify tissue perfusion. However, it could be used to more accurately estimate the arterial input function which is needed to compute the perfusion parameters [13]. Thus, it could be used in an ideal combination with the IS-PRI approach which can reconstruct the flow in vessels and tissue, but provides less accuracy when reconstructing the faster flow in vessels (Section III-E; cf. [8]).

Constrained image reconstruction can also be realized using the concepts of compressed sensing; this topic has recently gained considerable attention in the imaging community [34]. For example, prior image constrained compressed sensing (PICCS) is a technique to reconstruct time-resolved images [35]-[37]. In CT brain perfusion imaging, PICCS was investigated in order to reconstruct images using only a few projections (e.g., 4-20) distributed over the full view-angle range and thus saving X-ray dose [38]. Compared to the FBP reconstruction the results using PICCS showed significantly fewer streaking artifacts; however, in this study no perfusion values were investigated. In micro-CT imaging, PICCS has been used for noise reduction [39]. A decrease to $1 / 16$ of the noise variance of a FBP reconstruction was observed. Perfusion parameters maps were rated to have higher image quality when a PICCS reconstruction instead of a FBP reconstruction was used.

A further application of PICCS is to improve temporal resolution by using limited sector reconstruction (TRI-PICCS) [37], [40]-[42]. The projections are taken from a shorter viewangle range (e.g., $100^{\circ}$ ) than is necessary for traditional image reconstruction. TRI-PICCS has been investigated for cardiac CT imaging where an increase of the temporal resolution by a factor of 2-2.3 has been reported [41]. In the future, it may eventually be possible to apply this concept also to perfusion data acquired using a slow rotation C-arm CT system. In contrast to the 4-D vascular blood flow approach [33] tissue perfusion information could be obtained.

TRI-PICCS-based methods could potentially be applied to data acquired with an interleaved scanning protocol and be an alternative to the PRI approach. Eventually, the need for interleaving could even be relaxed and only one contrast bolus injection would be required. Topics for further research in the field of TRI-PICCS may include the resolution of lowcontrast changes due to the contrast agent flow in tissue; this is of particular importance and has not yet been investigated in detail. Furthermore, the computational time required by this iterative method must be sufficiently short such that it can be used during interventional stroke treatment.

\section{SUMMARY AND CONCLUSION}

We have developed a novel combined scanning and reconstruction approach that allows for tissue perfusion measurement in the interventional suite. Emphasis was put on computational speed of the methods, e.g. by using a modified FDK-based reconstruction algorithm, so that our technique could be used for intra-operative imaging during stroke therapy procedures.

Using numerical simulations we have shown that with two interleaved sequences the variability of estimated perfusion values is sufficiently low such that our method could be used for clinical decision making. These simulations were carried out with C-arm CT scan parameters that are representative of current systems (Table I). We expect that our results will improve with faster $\mathrm{C}$-arm rotation speeds accompanied by faster detector readout rates and shorter waiting times between rotations, e.g. realized with robotic $\mathrm{C}$-arm CT systems such as the Artis zeego (Siemens AG, Healthcare Sector, Forchheim, Germany). Furthermore, with new bi-plane C-arm angiography systems the two interleaved sequences could be acquired using a single contrast bolus injection only.

Our in vivo studies were based on a healthy pig model and the perfusion parameter maps computed from data acquired with our novel IS-PRI approach showed promising correlations with those from a reference perfusion CT. Further validation in stroke cases and human patient studies is necessary and will be carried out in the future.

In conclusion, this work is a novel and fundamental contribution towards perfusion imaging using $\mathrm{C}$-arm CT which could lead to optimized stroke treatment in the interventional suite.

\section{ACKNOWLEDGMENT}

This work was supported by a research grant from Siemens AG, Healthcare Sector, Forcheim, Germany. Financial support 
was also provided through NIH 1K99EB007676 and the Lucas Foundation. The authors gratefully acknowledge funding of the Erlangen Graduate School in Advanced Optical Technologies (SAOT) by the German Research Foundation (DFG) in the framework of the German excellence initiative. We thank Günter Lauritsch and Michael Manhart for helpful advice during preparation of the manuscript and Frank Dennerlein for providing parts of the simulation framework. The expert technical assistance of Wendy Baumgardner, Pamela Hertz and Lior Molvin is gratefully acknowledged.

\section{REFERENCES}

[1] R. G. González, J. A. Hirsch, W. J. Koroshetz, M. H. Lev, and P. W. Schaefer, Eds., Acute Ischemic Stroke, 1st ed. Berlin, Germany: Springer, 2006.

[2] A. Fieselmann, "Interventional perfusion imaging using C-arm computed tomography: Algorithms and clinical evaluation," Ph.D. dissertation, Friedrich-Alexander University of Erlangen-Nuremberg, Germany, 2011

[3] N. Strobel, O. Meissner, J. Boese, T. Brunner, B. Heigl, M. Hoheisel, G. Lauritsch, M. Nagel, M. Pfister, E.-P. Rührnschopf, B. Scholz, B. Schreiber, M. Spahn, M. Zellerhoff, and K. Klingenbeck-Regn, Multislice CT, 3rd ed. Berlin, Germany: Springer, 2009, ch. 3D Imaging with Flat-Detector C-Arm Systems, pp. 33-51.

[4] C. Neukirchen, M. Giordano, and S. Wiesner, "An iterative method for tomographic $\mathrm{x}$-ray perfusion estimation in a decomposition model-based approach," Medical Physics, vol. 37, no. 12, pp. 6125-6141, 2010.

[5] L. A. Feldkamp, L. C. Davis, and J. W. Kress, "Practical cone-beam algorithm," Journal of the Optical Society of America, vol. A1, pp. 612 619,1984

[6] C. Rohkohl, B. Keck, H. Hofmann, and J. Hornegger, "RabbitCT - an open platform for benchmarking 3D cone-beam reconstruction algorithms," Medical Physics, vol. 36, no. 9, pp. 3940-3944, 2009.

[7] P. Montes and G. Lauritsch, "A temporal interpolation approach for dynamic reconstruction in perfusion CT," Medical Physics, vol. 34 no. 7, pp. 3077-3092, 2007.

[8] A. Fieselmann, A. Ganguly, Y. Deuerling-Zheng, M. Zellerhoff, J. Boese, J. Hornegger, and R. Fahrig, "A dynamic reconstruction approach for cerebral blood flow quantification with an interventional C-arm CT," in Proc. IEEE International Symposium on Biomedical Imaging 2010, Rotterdam, The Netherlands, 2010, pp. 53-56.

[9] A. Ganguly, A. Fieselmann, M. Marks, J. Rosenberg, J. Boese, Y. Deuerling-Zheng, M. Straka, G. Zaharchuk, R. Bammer, and R. Fahrig, "Cerebral CT perfusion using an interventional C-arm imaging system: Cerebral blood flow measurements," American Journal of Neuroradiology, vol. 32, no. 8, pp. 1525-1531, 2011.

[10] G. Lauritsch, J. Boese, L. Wigström, H. Kemeth, and R. Fahrig, "Towards cardiac C-arm computed tomography," IEEE Transactions on Medical Imaging, vol. 25, no. 7, pp. 922-934, 2006.

[11] M. Wintermark, W. S. Smith, N. U. Ko, M. Quist, P. Schnyder, and W. P. Dillon, "Dynamic perfusion CT: optimizing the temporal resolution and contrast volume for calculation of perfusion CT parameters in stroke patients." American Journal of Neuroradiology, vol. 25, no. 5, pp. 720729, 2004.

[12] M. Wiesmann, S. Berg, G. Bohner, R. Klingebiel, V. Schöpf, B. M. Stoeckelhuber, I. Yousry, J. Linn, and U. Missler, "Dose reduction in dynamic perfusion CT of the brain: effects of the scan frequency on measurements of cerebral blood flow, cerebral blood volume, and mean transit time." European Radiology, vol. 18, no. 12, pp. 2967-2974, 2008.

[13] A. Fieselmann, M. Kowarschik, A. Ganguly, J. Hornegger, and R. Fahrig, "Deconvolution-based CT and MR brain perfusion measurement: Theoretical model revisited and practical implementation details," International Journal of Biomedical Imaging, vol. 2011, article ID 467563, 20 pages, 2011

[14] A. Fieselmann, F. Dennerlein, Y. Deuerling-Zheng, J. Boese, R. Fahrig, and J. Hornegger, "A model for filtered backprojection reconstruction artifacts due to time-varying attenuation values in perfusion C-arm CT," Physics in Medicine and Biology, vol. 56, no. 12, pp. 3701-3717, 2011.

[15] C. T. Badea, S. M. Johnston, E. Subashi, Y. Qi, L. W. Hedlund, and G. A. Johnson, "Lung perfusion imaging in small animals using 4D micro-CT at heartbeat temporal resolution," Medical Physics, vol. 37, no. 1, pp. 54-62, 2010.

[16] M. D. Silver, "A method for including redundant data in computed tomography," Medical Physics, vol. 27, no. 4, pp. 773-774, 2000.
[17] W. H. Press, S. A. Teukolsky, W. T. Vetterling, and B. P. Flannery, Numerical Recipes, 3rd ed. Cambridge, UK: Cambridge University Press, 2007.

[18] F. N. Fritsch and R. E. Carlson, "Monotone piecewise cubic interpolation," SIAM Journal on Numerical Analysis, vol. 17, pp. 238-246, 1980.

[19] M. D. Buhmann, Radial Basis Functions: Theory and Implementations, 1st ed. Cambridge, UK: Cambridge University Press, 2003.

[20] L. Østergaard, R. M. Weisskoff, D. A. Chesler, C. Gyldensted, and B. R. Rosen, "High resolution measurement of cerebral blood flow using intravascular tracer bolus passages. part I: Mathematical approach and statistical analysis," Magnetic Resonance in Medicine, vol. 36, no. 5, pp. 715-725, 1996.

[21] H. M. Silvennoinen, L. M. Hamberg, L. Valanne, and G. J. Hunter, "Increasing contrast agent concentration improves enhancement in firstpass CT perfusion," American Journal of Neuroradiology, vol. 28, no. 7 , pp. 1299-1303, 2007.

[22] J. Bredno, M. E. Olszewski, and M. Wintermark, "Simulation model for contrast agent dynamics in brain perfusion scans," Magnetic Resonance in Medicine, vol. 6, no. 1, pp. 280-290, 2010.

[23] A. Fieselmann, "DIPPO - a digital brain perfusion phantom," www5.cs.fau.de/ fieselma/data/, accessed December 20, 2011.

[24] A. Fieselmann, A. Ganguly, Y. Deuerling-Zheng, J. Boese, J. Hornegger, and R. Fahrig, "Automatic measurement of contrast bolus distribution in carotid arteries using a C-arm angiography system to support interventional perfusion imaging," in Proc. SPIE Medical Imaging 2011: Visualization, Image-Guided Procedures, and Modeling, vol. 7964, Lake Buena Vista, USA, 2011, pp. 79641W1-6.

[25] K. Kudo, M. Sasaki, K. Yamada, S. Momoshima, H. Utsunomiya, H. Shirato, and K. Ogasawara, "Differences in CT perfusion maps generated by different commercial software: Quantitative analysis by using identical source data of acute stroke patients," Radiology, vol. 254, no. 1, pp. 200-209, 2010.

[26] F. Zanderigo, A. Bertoldo, G. Pillonetto, and C. Cobelli, "Nonlinear stochastic regularization to characterize tissue residue function in bolustracking MRI: Assessment and comparison with SVD, block-circulant SVD, and Tikhonov," IEEE Transactions on Biomedical Engineering, vol. 56, no. 5, pp. 1287-1297, 2009.

[27] K. Kudo, S. Terae, C. Katoh, M. Oka, T. Shiga, N. Tamaki, and K. Miyasaka, "Quantitative cerebral blood flow measurement with dynamic perfusion CT using the vascular-pixel elimination method: Comparison with $\mathrm{H}_{2}^{15} \mathrm{O}$ positron emission tomography," American Journal of Neuroradiology, vol. 24, no. 3, pp. 419-426, 2003.

[28] P. Sauleaua, E. Lapoublea, D. Val-Lailleta, and C.-H. Malberta, "The pig model in brain imaging and neurosurgery," The International Journal of Animal Biosciences, vol. 3, no. 8, pp. 1138-1151, 2009.

[29] M. Mehra, N. Henninger, J. A. Hirsch, J. Chueh, A. K. Wakhloo, and M. J. Gounis, "Preclinical acute ischemic stroke modeling," Journal of NeuroInterventional Surgery, 2011, (in press).

[30] D. Morhard, C. D. Wirth, G. Fesl, C. Schmidt, M. F. Reiser, C. R. Becker, and B. Ertl-Wagner, "Advantages of extended brain perfusion computed tomography: $9.6 \mathrm{~cm}$ coverage with time resolved computed tomography-angiography in comparison to standard stroke-computed tomography," Investigative Radiology, vol. 45, no. 7, pp. 363-369, 2010.

[31] R. Fahrig, R. Dixon, T. Payne, R. Morin, A. Ganguly, and N. Strobel, "Dose and image quality for a cone-beam C-arm CT system," Medical Physics, vol. 33, no. 12, pp. 4541-50, 2006.

[32] C. A. Mistretta, E. Oberstar, B. Davis, E. Brodsky, and C. M. Strother, "4D-DSA and 4D fluoroscopy: preliminary implementation," in Proc. SPIE Medical Imaging 2010: Physics of Medical Imaging, vol. 7622, San Diego, USA, 2010, pp. 7622 271-8.

[33] C. A. Mistretta, "Sub-Nyquist acquisition and constrained reconstruction in time resolved angiography," Medical Physics, vol. 38, no. 6, pp. 29752985, 2011.

[34] M. Fornasier and H. Rauhut, Handbook of Mathematical Methods in Imaging, 1st ed. Berlin, Germany: Springer, 2011, ch. Compressive Sensing, pp. 187-228.

[35] G.-H. Chen, J. Tang, and S. Leng, "Prior image constrained compressed sensing (PICCS)," Proc. SPIE Photons Plus Ultrasound: Imaging and Sensing 2008, vol. 6856, p. 685618, 2008.

[36] _ "Prior image constrained compressed sensing (PICCS): a method to accurately reconstruct dynamic CT images from highly undersampled projection data sets," Medical Physics, vol. 35, no. 2, pp. 660-663, 2008.

[37] G.-H. Chen, J. Tang, B. Nett, Z. Qi, S. Leng, and T. Szczykutowicz, "Prior image constrained compressed sensing (PICCS) and applications in X-ray computed tomography,' Current Medical Imaging Reviews, vol. 6, no. 2, pp. 119-134, 2010. 
[38] J. C. Ramirez-Giraldo, J. Trzasko, S. Leng, L. Yu, A. Manduca, and C. H. McCollough, "Nonconvex prior image constrained compressed sensing (NCPICCS): Theory and simulations on perfusion CT," Medical Physics, vol. 38, no. 4, pp. 2157-2167, 2011.

[39] B. E. Nett, R. Brauweiler, W. Kalender, H. Rowley, and G.-H. Chen, "Perfusion measurements by micro-CT using prior image constrained compressed sensing (PICCS): initial phantom results," Physics in Medicine and Biology, vol. 21, no. 8, pp. 2333-2350, 2010.

[40] G.-H. Chen, J. Tang, and J. Hsieh, "Temporal resolution improvement using PICCS in MDCT cardiac imaging," Medical Physics, vol. 36, no. 6 , pp. 2130-2135, 2009.

[41] J. Tang, J. Hsieh, and G.-H. Chen, "Temporal resolution improvement in cardiac CT using PICCS (TRI-PICCS): performance studies," Medical Physics, vol. 37, no. 8, pp. 4377-4388, 2010.

[42] C. Maaß and M. Kachelrieß, "Quantification of temporal resolution and its reliability in the context of TRI-PICCS and dual source CT," in Proc. SPIE Medical Imaging 2011: Physics of Medical Imaging, vol. 7961, Lake Buena Vista, USA, 2011, pp. 79611M1-7. 